\title{
Electromagnetic Dissociation Cross Sections using Weisskopf-Ewing Theory
}

\author{
Anne M. Adamczyk ${ }^{1, *}$ and John W. Norbury ${ }^{2}$ \\ ${ }^{1}$ University of Tennessee \\ 315 Pasqua Engineering Building, Knoxville, TN, 37996-2300 \\ ${ }^{2} N A S A$ Langley Research Center \\ 2 West Reid Street, Bldg. 1205, Hampton, VA, 23681-2199
}

*Electronic address: aadamczy@utk.edu;

Number of Pages: 50;

Number of Tables: 3 ;

Number of Figures: 20 


\section{ABSTRACT}

It is important that accurate estimates of crew exposure to radiation are obtained for future long-term space missions. Presently, several space radiation transport codes exist to predict the radiation environment, all of which take as input particle interaction cross sections that describe the nuclear interactions between the particles and the shielding material. The space radiation transport code HZETRN uses the nuclear fragmentation model NUCFRG2 to calculate Electromagnetic Dissociation (EMD) cross sections. Currently, NUCFRG2 employs energy independent branching ratios to calculate these cross sections. Using Weisskopf-Ewing (WE) theory to calculate branching ratios, however, is more advantageous than the method currently employed in NUCFRG2. The WE theory can calculate not only neutron and proton emission, as in the energy independent branching ratio formalism used in NUCFRG2, but also deuteron, triton, helion, and alpha particle emission. These particles can contribute significantly to total exposure estimates. In this work, photonuclear cross sections are calculated using WE theory and the energy independent branching ratios used in NUCFRG2 and then compared to experimental data. It is found that the WE theory gives comparable, but mainly better agreement with data than the energy independent branching ratio. Furthermore, EMD cross sections for single neutron, proton, and alpha particle removal are calculated using WE theory and an energy independent branching ratio used in NUCFRG2 and compared to experimental data.

\section{INTRODUCTION}

Radiation protection is an important technology for the future of space exploration. When traveling into space, radiation is emitted from a variety of sources, such as the Van Allen 
trapped radiation belts, solar energetic particles, or galactic cosmic rays. Accurate estimates of the radiation exposure to astronauts or electronics is important for long term missions, such as the exploration of Mars. Several particle transport codes have been developed that estimate space radiation exposure. These transport codes take as input particle interaction cross sections that describe the nuclear interactions between the particles and the shielding material. Therefore, an accurate and efficient method of calculating cross sections is fundamental for shielding optimization.

A relativistic nucleus-nucleus collision will result when a cosmic ray nucleus interacts with a spacecraft wall or shielding material. Nucleus-nucleus collisions can be mediated by either the strong or electromagnetic (EM) forces. EM processes dominate when the collision's impact parameter is larger than the range of the nuclear force, so no nuclear interaction occurs. A reaction proceeding via the EM force is often called Electromagnetic Dissociation (EMD). For this type of reaction, a projectile nucleus is excited by the absorption of a virtual photon, which is supplied by the target nucleus. The projectile subsequently decays by the emission of one or more nucleons, with neutron emission the primary decay mode for nuclei with high atomic numbers, $Z$, and for photon energies below a few tens of $\mathrm{MeV}$. In the region of low $Z$ and energies above a few tens of $\mathrm{MeV}$, light ion production will compete with neutron emission. The Coulomb barrier suppresses light charged particle emission for heavy nuclei, but for lighter nuclei, the Coulomb barrier is lower, thus allowing for light ion production.

The high charge and energy (HZE) particle transport code HZETRN is a computational tool used for space radiation studies and shield design. HZETRN uses a deterministic approach for particle transport to enable fast and accurate estimates of the relevant dosimetric quantities $[1,2]$. HZETRN uses the nuclear fragmentation model NUCFRG2 [3] to calcu- 
late EMD cross sections. Currently, NUCFRG2 employs an energy independent branching ratio for the calculation of EMD nucleus-nucleus cross sections. This energy independent branching ratio can only account for neutron and proton emission. In this paper, a way of calculating EMD nucleus-nucleus cross sections, using an energy dependent branching ratio, is presented. This branching ratio can account for the emission of a neutron, proton, deuteron, triton, helion, and alpha particle, which is a significant improvement over the current NUCRFG2 branching ratio model. It should be noted that the additional ions have been shown to account for a non-negligible portion of the total exposure in some cases. The energy dependent branching ratio, which is calculated from Weisskopf-Ewing (WE) theory, is first folded into the photonuclear cross section formula and then incorporated into the EMD nucleus-nucleus cross section equation.

A large part of the uncertainty in an EMD nucleus-nucleus cross section calculation comes from the photonuclear cross section. The uncertainties generally arise due to the poor theoretical treatment of the branching ratio. This paper will compare an energy dependent branching ratio, which is calculated from WE theory, to the energy independent branching ratio currently employed by NUCFRG2. It is shown here that the energy dependent branching ratio, calculated with WE theory, provides better estimates of the photonuclear cross section, which consequently give more accurate EMD nucleus-nucleus cross sections. A review of the photonuclear cross section is first given, followed by a summary of the WE method and the energy independent branching ratio used in NUCFRG2. The EMD nucleus-nucleus cross section is also reviewed and comparisons are made. 


\section{PHOTONUCLEAR CROSS SECTION}

This paper focuses on photonuclear reactions where a photon interacts with a nucleus, typically at an energy above a few $\mathrm{MeV}$. When a nuclear excitation occurs, the main contribution comes from the Giant Dipole Resonance (GDR); the reaction results in the emission of neutrons, charged particles, or gamma rays. The photonuclear cross section is a fundamental component of an EMD nucleus-nucleus cross section, as it describes the response of the excited nucleus to spectator photons. If there is uncertainty in the photonuclear cross section, it will ultimately induce uncertainty into the EMD cross section. Therefore, it is important that the photonuclear cross section is well understood.

The photoparticle total cross section for producing particle $b$ is given by the product of the photonuclear absorption cross section, $\sigma_{\mathrm{abs}}\left(E_{\gamma}\right)$, and the branching ratio, $g_{b}$, representing the probability of a specific decay mode $[4,5]$;

$$
\sigma\left(E_{\gamma}, b\right)=g_{b} \sigma_{\mathrm{abs}}\left(E_{\gamma}\right)
$$

where $E_{\gamma}$ is the photon energy.

The branching ratio, $g_{b}$, will be discussed later, and it will be shown that the branching ratios can be modeled as either energy independent or energy dependent. Note that the branching ratio in equation (1) is energy independent; the dependence will be explicitly written if it is included.

Typically, experimental data are used for the absorption cross section, when available. For reactions with no experimental absorption cross section data, parameterizations are often used [5]. In addition, applications which require rapid cross section calculations, such 
as space radiation transport codes, employ a parameterized absorption cross section, since it would be impractical to put experimental photonuclear cross sections into EMD calculations for transport code analyses. The parameterized absorption cross section used in this paper will now be discussed.

\section{A. Absorption Cross Section}

The photonuclear absorption cross section, $\sigma_{\mathrm{abs}}\left(E_{\gamma}\right)$, is parameterized in the region near the giant dipole resonance as

$$
\sigma_{\mathrm{abs}}\left(E_{\gamma}\right)=\frac{\sigma_{m}}{1+\left[\left(E_{\gamma}^{2}-E_{\mathrm{GDR}}^{2}\right)^{2} / E_{\gamma}^{2} \Gamma^{2}\right]}
$$

The abbreviation, GDR, stands for the giant dipole resonance, as mentioned previously. Here, $E_{\mathrm{GDR}}$ is the energy at which the photonuclear cross section has its peak value, and $\Gamma$ is the width of the electric dipole (E1) giant dipole resonance. Values of $\Gamma$ used in this paper can be found in Table I. Also,

$$
\sigma_{m}=\frac{\sigma_{\mathrm{TRK}}}{\pi \Gamma / 2}
$$

with the Thomas-Reiche-Kuhn cross section given by [5]

$$
\sigma_{\mathrm{TRK}}=\frac{60 \mathcal{N}^{*} Z^{*}}{\mathcal{A}^{*}}
$$


with the ${ }^{*}$ referring to the excited nucleus. Here, $\mathcal{N}^{*}, Z^{*}$, and $\mathcal{A}^{*}$ are the neutron number, atomic number, and the mass number of the excited nucleus, respectively. The GDR energy is [5]

$$
E_{\mathrm{GDR}}=\frac{\hbar c}{\left[\frac{m_{0.7} c^{2} R_{0}^{2}}{8 J}\left(1+u-\frac{1+\epsilon+3 u}{1+\epsilon+u} \epsilon\right)\right]^{1 / 2}},
$$

with

$$
u=\frac{3 J}{Q^{\prime}} \mathcal{A}^{*-1 / 3}
$$

and

$$
R_{0}=r_{0} \mathcal{A}^{* 1 / 3}
$$

The parameters are [5]: 


$$
\begin{aligned}
\epsilon & =0.0768, \\
Q^{\prime} & =17 \mathrm{MeV}, \\
J & =36.8 \mathrm{MeV}, \\
r_{0} & =1.18 \mathrm{fm}, \\
m_{0.7} & =0.7 m_{\text {nucleon }},
\end{aligned}
$$

where $m_{\text {nucleon }}$ is the nucleon mass, taken as $938.95 \mathrm{MeV}$.

\section{BRANCHING RATIO}

A branching ratio provides the relative probability of a specific type of decay. In this section, a form for the branching ratio, which is dependent on the excitation energy of the compound nucleus, will be introduced that is calculated on the basis of the WE evaporation model of nuclear reactions. In addition, the energy independent branching ratio, used in NUCFRG2, will be presented. This energy independent branching ratio is not restricted to compound nucleus decay, but can also be applied to direct reactions.

\section{A. Energy Dependent Branching Ratio}

Weisskopf-Ewing (WE) theory is a commonly used method for computing an energy dependent branching ratio of a compound nucleus reaction. In a compound nucleus reaction, a compound nucleus is formed in an excited energy state and then decays into the products of the reaction once reaching statistical equilibrium. The formation and decay of the compound 
nucleus can be considered independent processes because only energy, angular momentum, and parity determine the de-excitation of the compound system.

The Hauser-Feshbach (HF) method provides another way of calculating cross sections for compound nucleus reactions. The difference between WE and HF lies in the number of allowable dynamical paths. The HF method accounts for more states, since parity and angular momentum are considered. The WE theory provides a simple way of calculating compound nuclear reactions going to continuum states, because only energy is conserved. The HF method has greater complexity than the WE theory and presents a much greater computational cost. Despite the fact that the HF method is generally favored for calculations of reactions proceeding through a compound nucleus state, the WE theory is chosen here due to its straightforward formulation and relatively low computational cost. Thus, the WE theory can be seen as a good alternative to the HF method for applications, such as radiation transport codes, that require near real time simulations. Here, computational efficiency must be weighed against the sensitivity of the results to the model.

The WE theory is developed by first considering a nuclear reaction where a particle $a$ strikes a nucleus $A$ to produce a residual nucleus $B$ and an outgoing particle $b$. The reaction proceeds though a well-defined compound state,

$$
\begin{gathered}
a+A \rightarrow C^{*} \rightarrow B+b \\
(\text { State } A) \quad(\text { State } C) \quad(\text { State } B)
\end{gathered}
$$

where $C$ represents the intermediate compound nucleus and the ${ }^{*}$ signifies an excited state. Particles $a$ and $b$ may be particles, such as photons, neutrons, and protons, although they 
could also be nuclei, such as deuterons, tritons, helions, or alpha particles. Note that a photonuclear reaction proceeds via $\gamma+A \rightarrow A^{*} \rightarrow B+b$.

The probability that the compound state will decay by emission of particle $b$ is given by the energy dependent branching ratio [6] as

$$
g_{b}\left(E_{C}^{*}\right)=\frac{\Gamma_{b}\left(E_{C}^{*}\right)}{\Gamma_{\text {tot }}\left(E_{C}^{*}\right)},
$$

where $\Gamma_{b}$ is the partial decay width for emitting particle $b, \Gamma_{\text {tot }}$ is the total width of the compound state, and $E_{C}^{*}$ is the excitation energy of the compound nucleus $C$. It is important to note that for a photonuclear reaction, the excitation energy will correspond to the photon energy, $E_{\gamma}$. Equation (14) will be referred to as the WE branching ratio. The total width is defined as the sum of all the partial widths. This work considers the emission of six particle types: neutron, proton, deuteron, triton, helion, and alpha. Emissions of other particles are assumed negligible. As a result, the total width is given by the sum of the six individual decay widths

$$
\Gamma_{\text {tot }}\left(E_{C}^{*}\right)=\Gamma_{n}\left(E_{C}^{*}\right)+\Gamma_{p}\left(E_{C}^{*}\right)+\Gamma_{d}\left(E_{C}^{*}\right)+\Gamma_{t}\left(E_{C}^{*}\right)+\Gamma_{h}\left(E_{C}^{*}\right)+\Gamma_{\alpha}\left(E_{C}^{*}\right),
$$

where $n, p, d, t, h$, and $\alpha$ represent a neutron, proton, deuteron, triton, helion, and alpha particle, respectively. Other decay modes, like fission and gamma emission, could also be included in equation (15) as additive terms. These decay modes have been discussed elsewhere [7, 8] and will not be discussed here. 
According to WE theory, the partial width of the decay in the numerator of equation (14) will be contingent on whether particle $b$ is charged. For the neutron, the partial width of decay is expressed as $[9,10]$

$$
\begin{aligned}
\Gamma_{n}\left(E_{C}^{*}\right) \approx & \frac{M_{n} C_{n}\left(2 s_{n}+1\right)}{\pi^{2} \hbar^{2}} \sigma_{\text {geo }} \frac{\rho_{B}\left(E_{C}^{*}-\epsilon_{n}\right)}{\rho_{C}\left(E_{C}^{*}\right)} T_{B} \\
& \times\left[\left(\epsilon_{n}-E_{C}^{*}-T_{B}-\beta_{n}\right) \exp \left(\frac{\epsilon_{n}-E_{C}^{*}}{T_{B}}\right)+T_{B}+\beta_{n}\right],
\end{aligned}
$$

where $M_{n}, s_{n}$, and $\epsilon_{n}$ are the mass, spin, and binding energy for the emitted neutron, respectively. Here, $\beta_{n}=\left(2.12 \mathcal{A}_{B}^{-2 / 3}-0.050\right) / C_{n}$ and $C_{n}=0.76+2.2 \mathcal{A}_{B}^{-1 / 3}$, where $\mathcal{A}_{B}$ is the atomic mass of the residual nucleus [11]. The geometrical cross section $\sigma_{\text {geo }}$, is represented by the classical target area, $\sigma_{\text {geo }}=\pi\left(r_{0} \mathcal{A}_{B}^{1 / 3}\right)^{2}$, where the radius parameter $r_{0}$ is taken to be $1.18 \mathrm{fm}[11]$. Also contained in equation (16) are the nuclear densities $\rho_{C}$ and $\rho_{B}$, for the compound nucleus $C$ and the residual nucleus $B$, respectively. See references $[12,13]$ for detailed information of all these terms.

The partial width for a charged particle is defined by $[9,10]$

$$
\begin{aligned}
\Gamma_{c}\left(E_{C}^{*}\right) \approx & \frac{M_{c}\left(2 s_{c}+1\right)}{\pi^{2} \hbar^{2}} \sigma_{\text {geo }} \frac{\rho_{B}\left(E_{C}^{*}-\epsilon_{c}\right)}{\rho_{C}\left(E_{C}^{*}\right)} T_{B} \\
& \times\left[\left(\epsilon_{c}-E_{C}^{*}-T_{B}-V_{c}\right) \times \exp \left(\frac{\epsilon_{c}-E_{C}^{*}}{T_{B}}\right)+T_{B} \exp \left(\frac{-V_{c}}{T_{B}}\right)\right],
\end{aligned}
$$

where $M_{c}, s_{c}, V_{c}$, and $\epsilon_{c}$, are the mass, spin, Coulomb barrier and binding energy for one of the charged particles $(p, d, t, h, \alpha)$, respectively.

Both of the expressions for the partial width contain $T_{B}$, the temperature of the residual 
nucleus after $b$ emission, which is calculated by $[9,10]$

$$
\begin{aligned}
T_{B}= & \left(\frac{d \ln \rho_{B}}{d E_{C}^{*}}\right)^{-1} \\
= & \left(\frac{E_{C}^{*}-\epsilon_{b}}{a_{B}\left(E_{C}^{*}-\epsilon_{b}\right)}\right)^{1 / 2}\left(1+\frac{\delta E_{0, B}}{E_{C}^{*}-\epsilon_{b}} \frac{\tilde{a}_{B}}{a_{B}\left(E_{C}^{*}-\epsilon_{b}\right)}\right. \\
& \left.\times\left[\exp \left(-\gamma_{B}\left[E_{C}^{*}-\epsilon_{b}\right]\right) \times\left(1+\gamma_{B}\left[E_{C}^{*}-\epsilon_{b}\right]\right)-1\right]\right)^{-1} .
\end{aligned}
$$

Here, $a_{B}, \tilde{a}_{B}, \delta E_{0, B}$, and $\gamma_{B}$ are the level density parameter, asymptotic level density parameter, shell correction energy, and damping parameter of the residual nucleus $B$, respectively. These terms and a complete derivation of the neutron and charged particle partial width formula, equations (16) and (17), have been discussed in detail in references [12, 13].

\section{B. Energy Independent Branching Ratio}

In the previous section, a method was introduced for calculating branching ratios, based on WE theory. This branching ratio formalism differs from the method used in NUCFRG2. Currently, NUCFRG2 employs a branching ratio from references [14, 15]. This branching ratio neglects energy dependence, unlike the WE theory, which takes into account the excitation energy (or for a photonuclear reaction, the photon energy). This energy independent branching ratio is not restricted to compound nucleus decay, but can also be applied to direct reactions. A direct reaction has a short interaction time, unlike the long lived intermediary state of a compound nucleus reaction. A compound nucleus reaction follows equation (13), but a direct reaction proceeds via 


$$
a+A \quad \rightarrow \quad B+b
$$

$($ State $A) \quad($ State $B)$

The energy dependent proton branching ratio utilized in NUCFRG2 is given by $[3,14,15]$

$$
g_{p}=\left\{\begin{array}{lc}
0.5 & Z_{A}^{*}<6 \\
0.6 & 6 \leq Z_{A}^{*} \leq 8 \\
0.7 & 8<Z_{A}^{*}<14 \\
\min \left[\frac{Z_{A}^{*}}{\mathcal{A}_{A}^{*}}, 1.95 \exp \left(-0.075 Z_{A}^{*}\right)\right] & 14 \leq Z_{A}^{*}
\end{array} .\right.
$$

Here, $Z_{A}^{*}$ and $\mathcal{A}_{A}^{*}$ are the atomic number and mass number of the excited nucleus $A$ (either the projectile or target), respectively. Due to the dependence on only the atomic number and mass number of the excited nucleus $A$, this energy independent branching ratio can be applied to a direct or compound nucleus reaction.

With the ratio of proton emission now formulated, the neutron branching ratio can be found easily from

$$
g_{n}=1-g_{p},
$$

since only proton and neutron decay are considered in NUCFRG2 [3]. For this crude approximation, emission of other particles was thought to compete insignificantly with proton and neutron emission, so other channels were neglected for simplicity. It should be noted that the above relations assume only single nucleon emission. 


\section{NUCLEUS-NUCLEUS CROSS SECTION}

In a nucleus-nucleus collision mediated by the EM force, the target (or projectile) represents a source of virtual photons, which impinge upon the projectile (or target). The spectrum of virtual photons contains a variety of energies, in contrast to a photonuclear reaction where the incoming photon possesses only a single energy.

The total cross section for electromagnetic nucleus-nucleus reactions can be written in the form

$$
\sigma_{A A}=\int d E_{\gamma} N\left(E_{\gamma}\right) \sigma_{\gamma A}\left(E_{\gamma}\right)
$$

where $N\left(E_{\gamma}\right)$ is the Weizsacker-Williams virtual photon spectrum and $\sigma_{\gamma A}\left(E_{\gamma}\right)$ is the photonuclear total cross section. The Weizsacker-Williams virtual photon spectrum will be discussed in the subsequent section.

Equation (1) expresses $\sigma_{\gamma A}\left(E_{\gamma}\right)$ as the branching ratio, $g_{X}$, multiplied by the absorption cross section, $\sigma_{\mathrm{abs}}\left(E_{\gamma}\right)$. When replacing the photonuclear total cross section in equation (22) with equation (1), the form of the total cross section for an electromagnetic nucleus-nucleus reaction will depend on whether the branching ratio is energy dependent or independent. For the case of an energy dependent branching ratio,

$$
\sigma_{A A}=\int d E_{\gamma} N\left(E_{\gamma}\right) g_{X}\left(E_{\gamma}\right) \sigma_{\mathrm{abs}}\left(E_{\gamma}\right)
$$

whereas an energy independent branching ratio can be brought outside the integral, 


$$
\sigma_{A A}=g_{X} \int d E_{\gamma} N\left(E_{\gamma}\right) \sigma_{\mathrm{abs}}\left(E_{\gamma}\right)
$$

which allows for faster computation.

\section{A. Weizsacker-Williams Virtual Photon Spectrum}

Equations (22), (23), and (24) require the Weizsacker-Williams virtual photon spectrum. The virtual photon spectrum describes the number of photons the target contributes at a specific energy and is given by $[16,17]$

$$
N\left(E_{\gamma}\right)=\frac{1}{E_{\gamma}} \frac{2}{\pi} Z_{S}^{2} \alpha_{\mathrm{fsc}} \frac{1}{\beta^{2}}\left[\xi K_{0}(\xi) K_{1}(\xi)-\frac{1}{2} \xi^{2} \beta^{2}\left(K_{1}^{2}(\xi)-K_{0}^{2}(\xi)\right)\right]
$$

where $Z_{S}$ is the nuclear charge of the spectator nucleus (either the projectile or target) and $\alpha_{\mathrm{fsc}}$ is the EM fine structure constant given by $\alpha_{\mathrm{fsc}}=e^{2} / \hbar c$. The relativistic beta factor is expressed as

$$
\beta=\sqrt{1-\frac{1}{\gamma^{\prime 2}}}
$$

and the relativistic gamma factor of the excited nucleus is given by 


$$
\gamma \prime=\frac{1+T_{\text {lab }}}{m_{\text {nucleon }}}
$$

where $T_{\text {lab }}$ is the kinetic energy per particle $N$ of the projectile. Equation (25) also contains the modified Bessel functions, $K_{0}(\xi)$ and $K_{1}(\xi)$, which are functions of the adiabacity parameter $\xi$;

$$
\xi=\frac{E_{\gamma} b_{\min }}{\gamma / \beta(\hbar c)}
$$

The minimum impact parameter, $b_{\text {min }}$, is the value below which the strong force is assumed to dominate [18] and is found from the relation [5]

$$
6_{\min }=R_{0.1, P}+R_{0.1, T}-d_{\text {overlap }}
$$

where $R_{0.1, P}$ and $R_{0.1, T}$ are the $10 \%$ charge density radii for the projectile and target nuclei, respectively. Values of the $10 \%$ charge density radii used in this paper can be found in Table II. The overlap distance is expressed as $d_{\text {overlap }}$ and is treated as an arbitrary parameter.

\section{COMPARISON TO EXPERIMENT}

The primary focus of this work was to evaluate and compare the WE branching ratio to the energy independent branching ratio, the method currently used in NUCFRG2, for the 
purposes of calculating EMD nucleus-nucleus cross sections. To obtain a true comparison of these branching ratios, the photonuclear cross section must be examined for reasons which will now be explained. Following this explanation, comparisons to experiment will be made for both branching ratio methods for EMD nucleus-nucleus cross sections.

\section{A. Photonuclear Cross Sections using WE and energy independent branching} ratios

Photonuclear cross sections, calculated with equation (1) are presented in this section. Comparisons to experimental data are made for a few nuclei, as shown in Figures 1 - 5. The photonuclear cross sections in this section are calculated using experimental absorption cross sections, from reference [19], at various photon energies. The experimental cross sections are multiplied by the WE branching ratio, given in equation (14), to produce the photonuclear cross sections shown in red in Figures 1 - 5. Similarly, the energy independent branching ratios are used, equations (20) and (21), to find the photonuclear cross section results shown in green.

By using experimental absorption cross sections, the WE and energy independent branching ratio can be compared directly, because it eliminates the possibility of induced uncertainty from a parameterized absorption cross section. The absorption cross section used in this paper, equation (2), for EMD nucleus-nucleus cross section comparisons is a Lorentzian parameterization. This absorption cross section will give the photonuclear cross section its dominant feature, a hump, which corresponds to the excitation of the giant dipole resonance. This parameterization will be poor for deformed nuclei, where the cross section is seen as the sum of two Lorentzians, and for light nuclei, which often exhibit complicated shapes $[20]$. 
It can be seen in Figures 1 - 5 that the theoretical photonuclear cross sections, calculated with WE and energy independent branching ratios, adequately agree with experiment. For most of the reactions, the theoretical photonuclear cross section, calculated with a WE branching ratio, provides better agreement to experiment. This can be observed for the reactions ${ }^{28} \operatorname{Si}(\gamma, \mathrm{n}),{ }^{28} \mathrm{Si}(\gamma, \mathrm{p}),{ }^{88} \mathrm{Sr}(\gamma, \mathrm{n})$, and ${ }^{91} \mathrm{Zr}(\gamma, \mathrm{n})$, as shown in Figures $2-5$, respectively. It should be noted that for Figures 4 and 5, the WE branching ratio provides a much better fit to the experimental values around the peak of the photonuclear cross section, therefore capturing the essential feature of the GDR.

For ${ }^{16} \mathrm{O}(\gamma, \mathrm{n})$, shown in Figure 1, it is unclear which branching ratio (WE or energy independent) provides the better fit to experimental results, without performing a statistical analysis. The WE theory will have problems evaluating reactions involving oxygen, since a significant contribution of the photonuclear reaction comes from direct reactions [19]. The WE theory is not intended to be used for reactions that proceed directly and do not pass through a well defined compound state.

Problems matching experimental data can be seen for reactions involving ${ }^{28} \mathrm{Si}$. The theoretical photoneutron and photoproton cross sections calculated using WE branching ratios seem to provide the better fit. Although, as seen in Figures 2 and 3, the WE branching ratio does not represent the data well. Reactions involving ${ }^{28} \mathrm{Si}$ are unique in the fact that compound nucleus decay is found for the neutron channel, but different behavior is observed for proton decay. Predominately direct Giant Dipole Resonance (GDR) decay occurs for the proton channels of ${ }^{28} \mathrm{Si}[21]$. Most of the partial (ground state, first excited state, etc.) proton cross sections demonstrate different intermediate structures [21]. Consequently, the branching ratios calculated with WE theory will not be representative of the proton decay channel. The erroneously calculated proton branching ratio will also have an effect on the 
other channels (neutron, deuteron, triton, helion, and alpha), because the denominator of the WE branching ratio formula contains the total width, which is given by the sum of all the decay channels. If one of the channels is calculated incorrectly, it will ultimately affect all of the channels.

From the comparisons presented, it is recommended that NUCFRG2 should use WE branching ratios rather than the energy independent method currently employed. For compound nucleus reactions, as in equation (13), the WE branching ratio provides the best

fit, which is shown for ${ }^{88} \operatorname{Sr}(\gamma, \mathrm{n})$ and ${ }^{91} \operatorname{Zr}(\gamma, \mathrm{n})$. For reactions that have single or multiple channels that proceed through a direct reaction, as in equation (19), the WE branching ratio provides the same or better results when compared to the energy independent branching ratio. This is surprising, since WE theory was not designed for reactions that proceed through direct channels. Due to the WE branching ratio giving similar, or better, results than the energy independent branching ratio, for direct and compound nucleus reactions, it is recommended that WE branching ratios be incorporated into NUCFRG2. In addition, the WE branching ratios are more advantageous, because they allow for not only neutron and proton emission, but also for deuteron, triton, helion, and alpha particle emission.

\section{B. Nucleus-Nucleus Cross Sections using WE and energy independent branching} ratios

In the previous section, a comparison of WE and energy independent branching ratios was presented for photonuclear cross sections. Conclusions made from the comparison and evaluation of photonuclear cross section results should be used for making future NUCFRG2 recommendations. This comparison eliminated the possibility of induced uncertainty from parameterized terms, as mentioned previously. In this section, EMD cross sections for 
single neutron, proton, and alpha particle removal are calculated using WE and energy independent branching ratios and then compared to experiment, as shown in Table III. Graphical depictions of this data are shown in Figures 6-20.

To calculate nucleus-nucleus cross sections using an energy independent branching ratio, equation (24) is employed, where the proton and neutron branching ratios are given by equations (20) and (21), respectively. It is important to reiterate that for the case of the energy independent branching ratio, the formulation was based on the assumption that only neutron and proton emission occurred. Therefore, single alpha particle removal EMD cross sections, calculated using energy independent branching ratios, could not be determined. In Table III, for single particle alpha particle removal, the results calculated with an energy independent branching ratio will be marked not applicable (N/A). The current capability to calculate only neutron and proton emission in NUCFRG2, due to energy independent branching ratios, makes WE theory very desirable.

For a nucleus-nucleus cross section that utilizes the WE branching ratio, equation (23) is used. The WE branching ratio is given by equation (14), with the total width given by equation (15) and the partial width given by equations (16) and (17) for a neutron and charged particle, respectively. Here, EMD cross sections for single alpha particle removal that employ WE branching ratios can be calculated, and are given in Table III.

Before comparing EMD nucleus-nucleus cross section results to experiment, using WE and energy independent branching ratios, it is necessary to consider a few caveats in this analysis. One is that EMD nucleus-nucleus cross sections are calculated with a parameterized absorption cross section. This parameterization is not intended for deformed nuclei, such as ${ }^{28} \mathrm{Si}[22]$ or for light nuclei, which exhibit complicated absorption cross section shapes. Another caveat is that there is very little experimental data concerning EMD nucleus-nucleus 
cross sections. Most experimental efforts have focused on special cases, in which the decay channel displays interesting behavior. Consequently, the experimental EMD nucleus-nucleus cross section data are not a good representation of a typical reaction. In addition, many of these experimental results have large errors, which demand better statistics for more accurate comparisons. It is important to consider all of these caveats when comparing EMD nucleus-nucleus cross section results, using WE and energy independent branching ratios, to experiment. For all the reasons listed above, the following results should not be used for making recommendations for NUCFGR2, but can offer insight into EMD nucleus-nucleus cross sections results that would be predicted by WE and energy independent branching ratios.

It can be seen that the EMD cross sections with decay channels ${ }^{11} \mathrm{C}+1 \mathrm{n}$ and ${ }^{11} \mathrm{~B}+$ $1 \mathrm{p}$, for a ${ }^{12} \mathrm{C}$ projectile, calculated using WE and energy independent branching ratios, are within the experimental error estimates for mostly all target and energy combinations, as shown in Figures 6-9. This also occurs with ${ }^{15} \mathrm{O}+1 \mathrm{n}$ and ${ }^{15} \mathrm{~N}+1 \mathrm{p}$ decay channels, for ${ }^{16} \mathrm{O}$ projectiles, as shown in Figures 10 and 11. It is surprising that the EMD cross sections calculated with WE branching ratios agree so well with the experimental data because ${ }^{12} \mathrm{C}$ and ${ }^{16} \mathrm{O}$ reactions proceed mainly through a direct reaction. The WE theory is specifically designed for compound nucleus decay and not intended for direct decay, as mentioned previously. Note that for the ${ }^{15} \mathrm{~N}+1 \mathrm{p}$ decay channel for a ${ }^{16} \mathrm{O}$ projectile has a negative experimental cross section, which is due to the large error bars.

It is obvious that discrepancies exist between the calculated EMD cross sections and experimental data for ${ }^{18} \mathrm{O}$ projectiles, as shown in Figures 12 and 13. The EMD cross sections calculated with WE and independent branching ratios will show disagreement because the parameterization of the absorption cross section, equation (2), was designed for only stable 
nuclei. Before comparisons can be made for ${ }^{18} \mathrm{O}$ projectiles, a parameterization for the absorption cross section for unstable nuclei will need to be developed.

Unexpected behavior can be observed for the ${ }^{28} \mathrm{Si}$ projectile. The EMD cross sections are calculated with a parameterized absorption cross section. As mentioned previously, this parameterization is not intended for a deformed nuclei, such as ${ }^{28} \mathrm{Si}$ [22]. It is surprising that the results predicted with a WE or energy independent branching ratio, give reasonable results for single neutron, proton, and alpha particle decay, as shown in Figures 14-16. In addition, WE results will be affected by the predominately direct GDR decay in the proton channel [21]. Therefore, the proton channel will be calculated incorrectly; this will ultimately effect the rest of the channels, as mentioned in the previous section.

Currently, NUCFRG2 does not have the capability of calculating alpha particle removal EMD cross sections. The energy independent branching ratio, currently employed in NUCFRG2, can calculate neutron and proton emission. In addition to neutron and proton removal, the WE branching ratio offers not only the calculation of alpha particle removal, but also deuteron, helion, and triton removal. The EMD cross sections for alpha particle removal of ${ }^{28} \mathrm{Si}$ on various targets, displayed in Figure 16, show results that are predicted by WE theory. It would be advantageous for NUCFRG2 to calculate additional channels, such as the alpha channel. Therefore, it is important to take a closer look at the predicted EMD cross sections for alpha particle removal. In Figure 16, it is observed that the theoretical cross sections, calculated with a WE branching ratio, compare well to experiment for ${ }^{27} \mathrm{Al},{ }^{64} \mathrm{Cu}$, and ${ }^{120} \mathrm{Sn}$ targets. Although, WE theory gives a much larger cross section than experimentally predicted for a ${ }^{208} \mathrm{~Pb}$ target. It is proposed here that this experimental EMD cross section for single alpha particle removal of ${ }^{28} \mathrm{Si}$ is too small. The scattering cross section should be proportional to the square of the atomic number of the 
target. Using the principle of detailed balance, a simple estimate of the EMD cross section for single alpha particle removal of ${ }^{28} \mathrm{Si}$ on a ${ }^{208} \mathrm{~Pb}$ target can be calculated. From the relation $\sigma_{208 P b} \propto\left(\frac{Z_{208 P b}}{Z_{*}}\right)^{2} \sigma_{*}$, where $\sigma_{208 P b}$ and $Z_{208 P b}$ are the cross section and atomic number for a ${ }^{208} \mathrm{~Pb}$ target, respectively. For $\sigma_{*}$ and $Z_{*}$, the cross section and atomic number values of ${ }^{27} \mathrm{Al},{ }^{64} \mathrm{Cu}$, and ${ }^{120} \mathrm{Sn}$ targets are used (the cross section data are taken from Table III). This simple and quick estimate gives a cross section value of approximately $836 \mathrm{mb}, 136$ $\mathrm{mb}$, and $156 \mathrm{mb}$ when using ${ }^{27} \mathrm{Al},{ }^{64} \mathrm{Cu}$, and ${ }^{120} \mathrm{Sn}$, respectively. The range of the estimated EMD alpha particle removal cross section of ${ }^{28} \mathrm{Si}$ on a ${ }^{208} \mathrm{~Pb}$ target is $136-836 \mathrm{mb}$, which is higher than the experimental value of $72 \pm 32 \mathrm{mb}$. It would be premature to invalidate the EMD alpha particle removal cross section of ${ }^{28} \mathrm{Si}$ on a ${ }^{208} \mathrm{~Pb}$ target, calculated with WE theory (264.33 mb), because its value lies well within the estimated range of 136-836 mb. Until better statistics or more experimental data becomes available, accurate comparisons cannot be made. Consequently, there is no reason to discredit using WE theory to calculate alpha particle removal EMD cross sections.

Problems calculating EMD cross sections with WE branching ratios can be observed for the ${ }^{88} \mathrm{Y}+1 \mathrm{n}$ decay channel, Figure 17 . This occurs for every projectile and energy combination. The energy independent branching ratio offers reasonable estimates, while the WE branching ratio experiences problems. Complications arise in ${ }^{89} \mathrm{Y}$ because $75 \%$ of the photoproton cross section between 14 and $24 \mathrm{MeV}$ decays directly [23-25]. The partial direct decay behavior in the proton channel will not be accounted for by the WE theory, which calculates decay rates for compound nucleus reactions. Just as in the case of ${ }^{28}$ Si projectiles, the erroneously calculated proton channel will affect all of the other decay channels.

For the decay channels, ${ }^{58} \mathrm{Co}+1 \mathrm{n}$ and ${ }^{197} \mathrm{Au}+1 \mathrm{n}$, the WE and energy independent branching ratios give similar results for mostly all of the projectile and energy combinations, 
Figures 18 and 19-20, respectively. It is important to note that out of all the EMD cross sections in this work, these two decay channels are the only channels that follow compound nucleus decay and do not have one or more channels that follow direct decay. This means that these are the only decay channels in this analysis that the WE theory is intended to apply. Both the WE and energy independent branching ratio calculate EMD cross sections, for the ${ }^{58} \mathrm{Co}+1 \mathrm{n}$ and ${ }^{197} \mathrm{Au}+1 \mathrm{n}$ decay channels, that agree reasonably well with experiment.

\section{CONCLUSION}

The nuclear fragmentation model NUCFRG2 currently calculates EMD nucleus-nucleus cross sections using energy independent branching ratios. Results indicate that the WE theory provides photonuclear cross sections that are comparable, but in most cases, better than the results obtained with energy independent branching ratios. Consequently, the WE theory should be considered as a good alternative to the energy independent branching ratio, which is currently employed in NUCFRG2. It was argued that the NUCFRG2 model should incorporate WE theory for the calculation of EMD cross sections and should consider the sensitivity of the results over computational time. The energy independent branching ratio allows for faster computation times of EMD nucleus-nucleus cross sections, due to its energy independence and simple formalism. The ability of the WE branching ratio to calculate not only neutron and proton emission, but also deuteron, triton, helion, and alpha particle emission make WE theory more advantageous.

Future work will include the investigation of a branching ratio formalism that can be applied to direct channels for future implementation into NUCFRG2. 
TABLE I: Values for the width of the electric dipole giant dipole resonance.

\begin{tabular}{ccc}
\hline \hline Nucleus & $\Gamma(\mathrm{MeV})$ & Reference \\
\hline${ }^{12} \mathrm{C}$ & 8.0 & {$[5]$} \\
${ }^{16} \mathrm{O}$ & 10.0 & {$[5]$} \\
${ }^{18} \mathrm{O}$ & 12.0 & {$[5]$} \\
${ }^{28} \mathrm{Si}$ & 10.0 & {$[5]$} \\
${ }^{59} \mathrm{Co}$ & 9.0 & {$[26]$} \\
${ }^{89} \mathrm{Y}$ & 3.95 & {$[27]$} \\
${ }^{197} \mathrm{Au}$ & 3.5 & {$[5]$} \\
\hline \hline
\end{tabular}


TABLE II: Values for the 10\% Charge Density Radius found from various models.

\begin{tabular}{ccc}
\hline \hline & & \\
Nucleus & $10 \%$ Chage Radius (fm) & Reference \\
\hline${ }^{12} \mathrm{C}$ & 3.33 & {$[5]$} \\
${ }^{16} \mathrm{O}$ & 3.77 & {$[5]$} \\
${ }^{18} \mathrm{O}$ & 3.88 & {$[5]$} \\
${ }^{20} \mathrm{Ne}$ & 4.06 & {$[5]$} \\
${ }^{27} \mathrm{Al}$ & 4.21 & {$[5]$} \\
${ }^{28} \mathrm{Si}$ & 4.18 & {$[5]$} \\
${ }^{32} \mathrm{~S}$ & 4.53 & {$[5]$} \\
${ }^{48} \mathrm{Ti}$ & 5.00 & {$[5]$} \\
${ }^{56} \mathrm{Fe}$ & 5.28 & {$[5]$} \\
${ }^{59} \mathrm{Co}$ & 5.33 & {$[28]$} \\
${ }^{64} \mathrm{Cu}$ & 5.45 & {$[5]$} \\
${ }^{89} \mathrm{Y}$ & 6.02 & {$[28]$} \\
${ }^{108} \mathrm{Ag}$ & 6.32 & {$[5]$} \\
${ }^{120} \mathrm{Sn}$ & 6.58 & {$[28]$} \\
${ }^{139} \mathrm{La}$ & 6.89 & {$[5]$} \\
${ }^{197} \mathrm{Au}$ & 7.56 & {$[5]$} \\
${ }^{208} \mathrm{~Pb}$ & 7.83 & {$[28]$} \\
${ }^{209} \mathrm{Bi}$ & 7.78 & {$[5]$} \\
${ }^{238} \mathrm{U}$ & 8.13 & \\
\hline \hline
\end{tabular}


TABLE III: Electromagnetic Cross Sections for Single Neutron, Proton, and Alpha Particle Removal. $\sigma_{\text {expt }}^{\text {EMD }}$ are the experimental EMD cross sections from references [30-36]. $\sigma_{(24)}^{\mathrm{EMD}}$ are the theoretical EMD cross sections using the energy independent branching ratio, equation $(24) . \sigma_{(23)}^{\mathrm{EMD}}$ are the theoretical EMD cross sections using a WE branching ratio, equation (23). Note that all data from this table are graphically displayed in Figures 6-20.

Projectile Target $\mathrm{T}_{\text {lab }}(\mathrm{GeV} / \mathrm{N})$ Decay Channel $\sigma_{\text {expt }}^{\mathrm{EMD}}(\mathrm{mb}) \sigma_{(24)}^{\mathrm{EMD}}(\mathrm{mb}) \sigma_{(23)}^{\mathrm{EMD}}(\mathrm{mb})$

\begin{tabular}{|c|c|c|c|c|c|c|}
\hline${ }^{12} \mathrm{C}$ & ${ }^{208} \mathrm{~Pb}$ & 2.1 & ${ }^{11} \mathrm{C}+1 \mathrm{n}$ & $51 \pm 18$ & 42.9964 & 35.2666 \\
\hline${ }^{12} \mathrm{C}$ & ${ }^{208} \mathrm{~Pb}$ & 2.1 & ${ }^{11} \mathrm{~B}+1 \mathrm{p}$ & $50 \pm 25$ & 70.2699 & 38.6367 \\
\hline${ }^{12} \mathrm{C}$ & ${ }^{208} \mathrm{~Pb}$ & 1.05 & ${ }^{11} \mathrm{C}+1 \mathrm{n}$ & $39 \pm 24$ & 25.2166 & 19.8329 \\
\hline${ }^{12} \mathrm{C}$ & ${ }^{208} \mathrm{~Pb}$ & 1.05 & ${ }^{11} \mathrm{~B}+1 \mathrm{p}$ & $50 \pm 25$ & 41.8808 & 22.1407 \\
\hline${ }^{16} \mathrm{O}$ & ${ }^{208} \mathrm{~Pb}$ & 2.1 & ${ }^{15} \mathrm{O}+1 \mathrm{n}$ & $50 \pm 24$ & 62.051 & 50.2401 \\
\hline${ }^{16} \mathrm{O}$ & ${ }^{208} \mathrm{~Pb}$ & 2.1 & ${ }^{15} \mathrm{~N}+1 \mathrm{p}$ & $96 \pm 26$ & 102.91 & 83.5714 \\
\hline${ }^{12} \mathrm{C}$ & ${ }^{108} \mathrm{Ag}$ & 2.1 & ${ }^{11} \mathrm{C}+1 \mathrm{n}$ & $21 \pm 10$ & 16.7542 & 13.8676 \\
\hline${ }^{12} \mathrm{C}$ & ${ }^{108} \mathrm{Ag}$ & 2.1 & ${ }^{11} \mathrm{~B}+1 \mathrm{p}$ & $18 \pm 13$ & 27.2921 & 15.1292 \\
\hline${ }^{12} \mathrm{C}$ & ${ }^{108} \mathrm{Ag}$ & 1.05 & ${ }^{11} \mathrm{C}+1 \mathrm{n}$ & $21 \pm 10$ & 10.5761 & 8.44344 \\
\hline${ }^{12} \mathrm{C}$ & ${ }^{108} \mathrm{Ag}$ & 1.05 & ${ }^{11} \mathrm{~B}+1 \mathrm{p}$ & $25 \pm 19$ & 17.4608 & 9.36414 \\
\hline${ }^{16} \mathrm{O}$ & ${ }^{108} \mathrm{Ag}$ & 2.1 & ${ }^{15} \mathrm{O}+1 \mathrm{n}$ & $26 \pm 13$ & 23.9174 & 19.532 \\
\hline${ }^{16} \mathrm{O}$ & ${ }^{108} \mathrm{Ag}$ & 2.1 & ${ }^{15} \mathrm{~N}+1 \mathrm{p}$ & $30 \pm 16$ & 39.4881 & 32.1429 \\
\hline${ }^{12} \mathrm{C}$ & ${ }^{64} \mathrm{Cu}$ & 2.1 & ${ }^{11} \mathrm{C}+1 \mathrm{n}$ & $10 \pm 7$ & 7.06394 & 5.87764 \\
\hline${ }^{12} \mathrm{C}$ & ${ }^{64} \mathrm{Cu}$ & 2.1 & ${ }^{11} \mathrm{~B}+1 \mathrm{p}$ & $4 \pm 8$ & 11.4855 & 6.39671 \\
\hline${ }^{12} \mathrm{C}$ & ${ }^{64} \mathrm{Cu}$ & 1.05 & ${ }^{11} \mathrm{C}+1 \mathrm{n}$ & $9 \pm 8$ & 4.65276 & 3.74663 \\
\hline${ }^{12} \mathrm{C}$ & ${ }^{64} \mathrm{Cu}$ & 1.05 & ${ }^{11} \mathrm{~B}+1 \mathrm{p}$ & $5 \pm 8$ & 7.65609 & 4.13926 \\
\hline${ }^{16} \mathrm{O}$ & ${ }^{64} \mathrm{Cu}$ & 2.1 & ${ }^{15} \mathrm{O}+1 \mathrm{n}$ & $9 \pm 8$ & 10.0187 & 8.2224 \\
\hline${ }^{16} \mathrm{O}$ & ${ }^{64} \mathrm{Cu}$ & 2.1 & ${ }^{15} \mathrm{~N}+1 \mathrm{p}$ & $15 \pm 8$ & 16.499 & 13.4477 \\
\hline${ }^{12} \mathrm{C}$ & ${ }^{27} \mathrm{Al}$ & 2.1 & ${ }^{11} \mathrm{C}+1 \mathrm{n}$ & $0 \pm 5$ & 1.6516 & 1.3846 \\
\hline${ }^{12} \mathrm{C}$ & ${ }^{27} \mathrm{Al}$ & 2.1 & ${ }^{11} \mathrm{~B}+1 \mathrm{p}$ & $0 \pm 5$ & 2.67839 & 1.50157 \\
\hline${ }^{12} \mathrm{C}$ & ${ }^{27} \mathrm{Al}$ & 1.05 & ${ }^{11} \mathrm{C}+1 \mathrm{n}$ & $1 \pm 6$ & 1.15605 & 0.942364 \\
\hline${ }^{12} \mathrm{C}$ & ${ }^{27} \mathrm{Al}$ & 1.05 & ${ }^{11} \mathrm{~B}+1 \mathrm{p}$ & $1 \pm 7$ & 1.89353 & 1.03538 \\
\hline${ }^{16} \mathrm{O}$ & ${ }^{27} \mathrm{Al}$ & 2.1 & ${ }^{15} \mathrm{O}+1 \mathrm{n}$ & $0 \pm 5$ & 2.31977 & 1.91739 \\
\hline${ }^{16} \mathrm{O}$ & ${ }^{27} \mathrm{Al}$ & 2.1 & ${ }^{15} \mathrm{~N}+1 \mathrm{p}$ & $-1 \pm 9$ & 3.80662 & 3.10831 \\
\hline
\end{tabular}

Continued on Next Page... 
Projectile Target $\mathrm{T}_{\text {lab }}(\mathrm{GeV} / \mathrm{N})$ Decay Channel $\sigma_{\text {expt }}^{\text {EMD }}(\mathrm{mb}) \sigma_{(24)}^{\text {EMD }}(\mathrm{mb}) \sigma_{(23)}^{\text {EMD }}(\mathrm{mb})$

$\begin{array}{lccllll}{ }^{12} \mathrm{C} & { }^{12} \mathrm{C} & 2.1 & { }^{11} \mathrm{C}+1 \mathrm{n} & -2 \pm 5 & 0.393841 & 0.331945 \\ { }^{12} \mathrm{C} & { }^{12} \mathrm{C} & 2.1 & { }^{11} \mathrm{~B}+1 \mathrm{p} & -1 \pm 4 & 0.637513 & 0.359076 \\ { }^{12} \mathrm{C} & { }^{12} \mathrm{C} & 1.05 & { }^{11} \mathrm{C}+1 \mathrm{n} & -2 \pm 5 & 0.287878 & 0.236711 \\ { }^{12} \mathrm{C} & { }^{12} \mathrm{C} & 1.05 & { }^{11} \mathrm{~B}+1 \mathrm{p} & -2 \pm 5 & 0.470029 & 0.259044 \\ { }^{16} \mathrm{O} & { }^{12} \mathrm{C} & 2.1 & { }^{15} \mathrm{O}+1 \mathrm{n} & -1 \pm 4 & 0.549095 & 0.456151 \\ { }^{16} \mathrm{O} & { }^{12} \mathrm{C} & 2.1 & { }^{15} \mathrm{~N}+1 \mathrm{p} & -1 \pm 4 & 0.898782 & 0.734837\end{array}$

$\begin{array}{ccccccc}{ }^{18} \mathrm{O} & { }^{238} \mathrm{U} & 1.7 & { }^{17} \mathrm{O}+1 \mathrm{n} & 140.8 \pm 4.1 & 87.5476 & 211.602 \\ { }^{18} \mathrm{O} & { }^{238} \mathrm{U} & 1.7 & { }^{17} \mathrm{~N}+1 \mathrm{p} & 25.1 \pm 1.6 & 101.782 & 0.419803 \\ { }^{18} \mathrm{O} & { }^{208} \mathrm{~Pb} & 1.7 & { }^{17} \mathrm{O}+1 \mathrm{n} & 136 \pm 2.9 & 71.7717 & 173.512 \\ { }^{18} \mathrm{O} & { }^{208} \mathrm{~Pb} & 1.7 & { }^{17} \mathrm{~N}+1 \mathrm{p} & 20.2 \pm 1.8 & 83.6706 & 0.34833 \\ { }^{18} \mathrm{O} & { }^{48} \mathrm{Ti} & 1.7 & { }^{17} \mathrm{O}+1 \mathrm{n} & 8.7 \pm 2.7 & 7.05503 & 17.0903 \\ { }^{18} \mathrm{O} & { }^{48} \mathrm{Ti} & 1.7 & { }^{17} \mathrm{~N}+1 \mathrm{p} & -0.5 \pm 1.0 & 8.43365 & 0.0383709 \\ & & & & & \\ & & & & & \\ { }^{28} \mathrm{Si} & { }^{208} \mathrm{~Pb} & 14.6 & { }^{27} \mathrm{Si}+1 \mathrm{n} & 241.0 \pm 4.2 & 409.25 & 153.513 \\ \ldots & \ldots & \ldots & \ldots & 263.5 \pm 15 & \ldots & \ldots \\ { }^{28} \mathrm{Si} & { }^{208} \mathrm{~Pb} & 14.6 & { }^{27} \mathrm{Al}+1 \mathrm{p} & 676.4 \pm 7.6 & 516.844 & 646.874 \\ \ldots & \ldots & \ldots & \ldots & 672.2 \pm 25 & \ldots & \ldots \\ { }^{28} \mathrm{Si} & { }^{208} \mathrm{~Pb} & 14.6 & { }^{24} \mathrm{Mg}+1 \alpha & 72 \pm 32 & \mathrm{~N} / \mathrm{A} & 264.33\end{array}$

$\begin{array}{ccccccc}{ }^{28} \mathrm{Si} & { }^{120} \mathrm{Sn} & 14.6 & { }^{27} \mathrm{Si}+1 \mathrm{n} & 100.0 \pm 2.0 & 160.077 & 60.1917 \\ \ldots & \ldots & \ldots & \ldots & 101.9 \pm 8.2 & \ldots & \ldots \\ { }^{28} \mathrm{Si} & { }^{120} \mathrm{Sn} & 14.6 & { }^{27} \mathrm{Al}+1 \mathrm{p} & 274.0 \pm 4.4 & 201.801 & 252.733 \\ \ldots & \ldots & \ldots & \ldots & 290.6 \pm 13 & \ldots & \ldots \\ { }^{28} \mathrm{Si} & { }^{120} \mathrm{Sn} & 14.6 & { }^{24} \mathrm{Mg}+1 \alpha & 58 \pm 30 & \mathrm{~N} / \mathrm{A} & 102.647\end{array}$

$\begin{array}{lcccccc}{ }^{28} \mathrm{Si} & { }^{64} \mathrm{Cu} & 14.6 & { }^{27} \mathrm{Si}+1 \mathrm{n} & 40.4 \pm 1.7 & 56.5488 & 21.3114 \\ { }^{28} \mathrm{Si} & { }^{64} \mathrm{Cu} & 14.6 & { }^{27} \mathrm{Al}+1 \mathrm{p} & 111.0 \pm 3.3 & 71.17 & 89.1858 \\ { }^{28} \mathrm{Si} & { }^{64} \mathrm{Cu} & 14.6 & { }^{24} \mathrm{Mg}+1 \alpha & 17 \pm 19 & \text { N/A } & 36.0171\end{array}$

$\begin{array}{ccccccc}{ }^{28} \mathrm{Si} & { }^{27} \mathrm{Al} & 14.6 & { }^{27} \mathrm{Si}+1 \mathrm{n} & 13.11 \pm 0.59 & 12.0398 & 4.54909 \\ \ldots & \ldots & \ldots & \ldots & 10.8 \pm 2.8 & \ldots & \ldots \\ { }^{28} \mathrm{Si} & { }^{27} \mathrm{Al} & 14.6 & 27 \mathrm{Al}+1 \mathrm{p} & 31.6 \pm 1.2 & 15.1243 & 18.9656 \\ \ldots & \ldots & \ldots & \ldots & 34.2 \pm 4.7 & \ldots & \ldots \\ { }^{28} \mathrm{Si} & { }^{27} \mathrm{Al} & 14.6 & { }^{24} \mathrm{Mg}+1 \alpha & 21 \pm 16 & \mathrm{~N} / \mathrm{A} & 7.6096\end{array}$

Continued on Next Page... 
Projectile Target $\mathrm{T}_{\text {lab }}(\mathrm{GeV} / \mathrm{N})$ Decay Channel $\sigma_{\operatorname{expt}}^{\mathrm{EMD}}(\mathrm{mb}) \sigma_{(24)}^{\mathrm{EMD}}(\mathrm{mb}) \sigma_{(23)}^{\mathrm{EMD}}(\mathrm{mb})$

$\begin{array}{ccccccc}{ }^{12} \mathrm{C} & { }^{197} \mathrm{Au} & 2.1 & { }^{196} \mathrm{Au}+1 \mathrm{n} & 75 \pm 4 & 36.9002 & 34.2595 \\ { }^{12} \mathrm{C} & { }^{89} \mathrm{Y} & 2.1 & { }^{88} \mathrm{Y}+1 \mathrm{n} & 9 \pm 12 & 11.9561 & 0.152798 \\ { }^{12} \mathrm{C} & { }^{59} \mathrm{Co} & 2.1 & { }^{58} \mathrm{Co}+1 \mathrm{n} & 6 \pm 9 & 5.37801 & 3.21086 \\ { }^{16} \mathrm{O} & { }^{197} \mathrm{Au} & 60 & { }^{196} \mathrm{Au}+1 \mathrm{n} & 280 \pm 30 & 214.465 & 201.668 \\ { }^{16} \mathrm{O} & { }^{197} \mathrm{Au} & 200 & { }^{196} \mathrm{Au}+1 \mathrm{n} & 440 \pm 40 & 278.427 & 262.135 \\ & & & & & \\ { }^{20} \mathrm{Ne} & { }^{197} \mathrm{Au} & 1.7 & { }^{196} \mathrm{Au}+1 \mathrm{n} & 151 \pm 13 & 97.2338 & 90.2015 \\ { }^{20} \mathrm{Ne} & { }^{197} \mathrm{Au} & 2.1 & { }^{196} \mathrm{Au}+1 \mathrm{n} & 153 \pm 18 & 88.6733 & 82.084 \\ { }^{20} \mathrm{Ne} & { }^{89} \mathrm{Y} & 2.1 & { }^{88} \mathrm{Y}+1 \mathrm{n} & 43 \pm 12 & 31.1781 & 0.392326 \\ { }^{20} \mathrm{Ne} & { }^{59} \mathrm{Co} & 2.1 & { }^{58} \mathrm{Co}+1 \mathrm{n} & 32 \pm 11 & 13.9518 & 8.3017 \\ & & & & & & \\ { }^{40} \mathrm{Ar} & { }^{197} \mathrm{Au} & 1.8 & { }^{196} \mathrm{Au}+1 \mathrm{n} & 348 \pm 34 & 279.913 & 259.048 \\ { }^{40} \mathrm{Ar} & { }^{89} \mathrm{Y} & 1.8 & { }^{88} \mathrm{Y}+1 \mathrm{n} & 132 \pm 17 & 88.5417 & 1.06924 \\ & & & & & & \\ { }^{56} \mathrm{Fe} & { }^{197} \mathrm{Au} & 1.7 & { }^{196} \mathrm{Au}+1 \mathrm{n} & 601 \pm 54 & 545.735 & 504.356 \\ { }^{56} \mathrm{Fe} & { }^{89} \mathrm{Y} & 1.7 & { }^{88} \mathrm{Y}+1 \mathrm{n} & 217 \pm 20 & 170.922 & 2.01472 \\ { }^{56} \mathrm{Fe} & { }^{59} \mathrm{Co} & 1.7 & { }^{58} \mathrm{Co}+1 \mathrm{n} & 88 \pm 14 & 75.779 & 44.447 \\ & & & & & \\ { }^{139} \mathrm{La} & { }^{197} \mathrm{Au} & 0.15 & { }^{196} \mathrm{Au}+1 \mathrm{n} & 447 \pm 28 & 604.729 & 510.793 \\ { }^{139} \mathrm{La} & { }^{197} \mathrm{Au} & 1.26 & { }^{196} \mathrm{Au}+1 \mathrm{n} & 1970 \pm 130 & 1994.08 & 1830.42 \\ { }^{139} \mathrm{La} & { }^{59} \mathrm{Co} & 1.26 & { }^{58} \mathrm{Co}+1 \mathrm{n} & 280 \pm 40 & 263.184 & 150.123 \\ & & & & & & \\ { }^{32} \mathrm{~S} & { }^{197} \mathrm{Au} & 200 & { }^{196} \mathrm{Au}+1 \mathrm{n} & 1120 \pm 160 & 1099.77 & 1035.37 \\ { }^{197} \mathrm{Au} & { }^{197} \mathrm{Au} & 1.0 & { }^{196} \mathrm{Au}+1 \mathrm{n} & 3077 \pm 200 & 3207.16 & 2927.37 \\ { }^{209} \mathrm{Bi} & { }^{197} \mathrm{Au} & 1.0 & { }^{196} \mathrm{Au}+1 \mathrm{n} & 3244 \pm 205 & 3469.23 & 3165.08 \\ { }^{197} \mathrm{Au} & 0.96 & { }^{196} \mathrm{Au}+1 \mathrm{n} & 3160 \pm 230 & 4037.1 & 3677.04\end{array}$




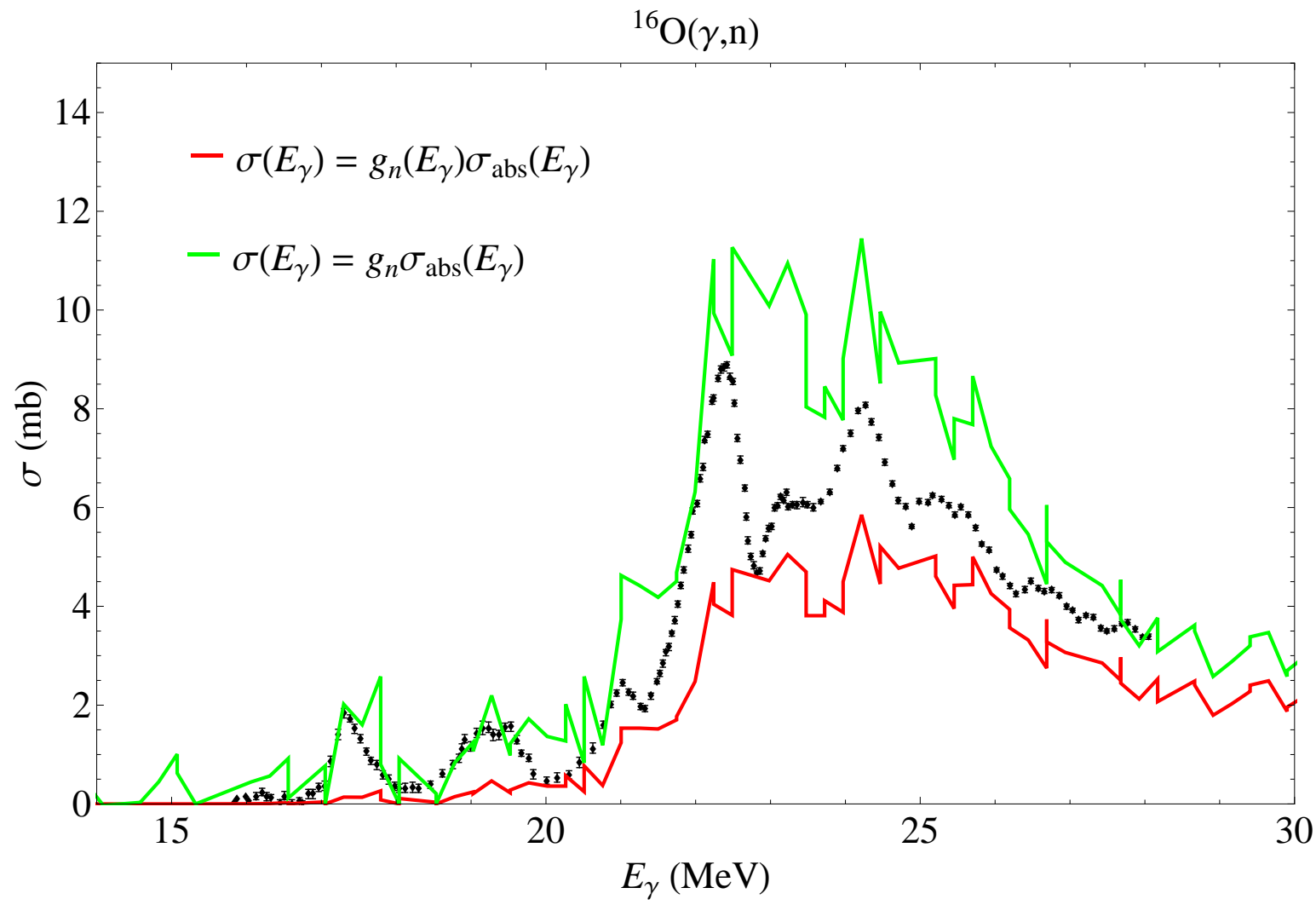

FIG. 1: Comparison of theoretical and experimental photoneutron cross sections for ${ }^{16} \mathrm{O}$. The theoretical photoneutron cross sections are calculated using WE (red) and energy independent branching ratios (green). Experimental data are from Figure 14(c) of reference [27]. 


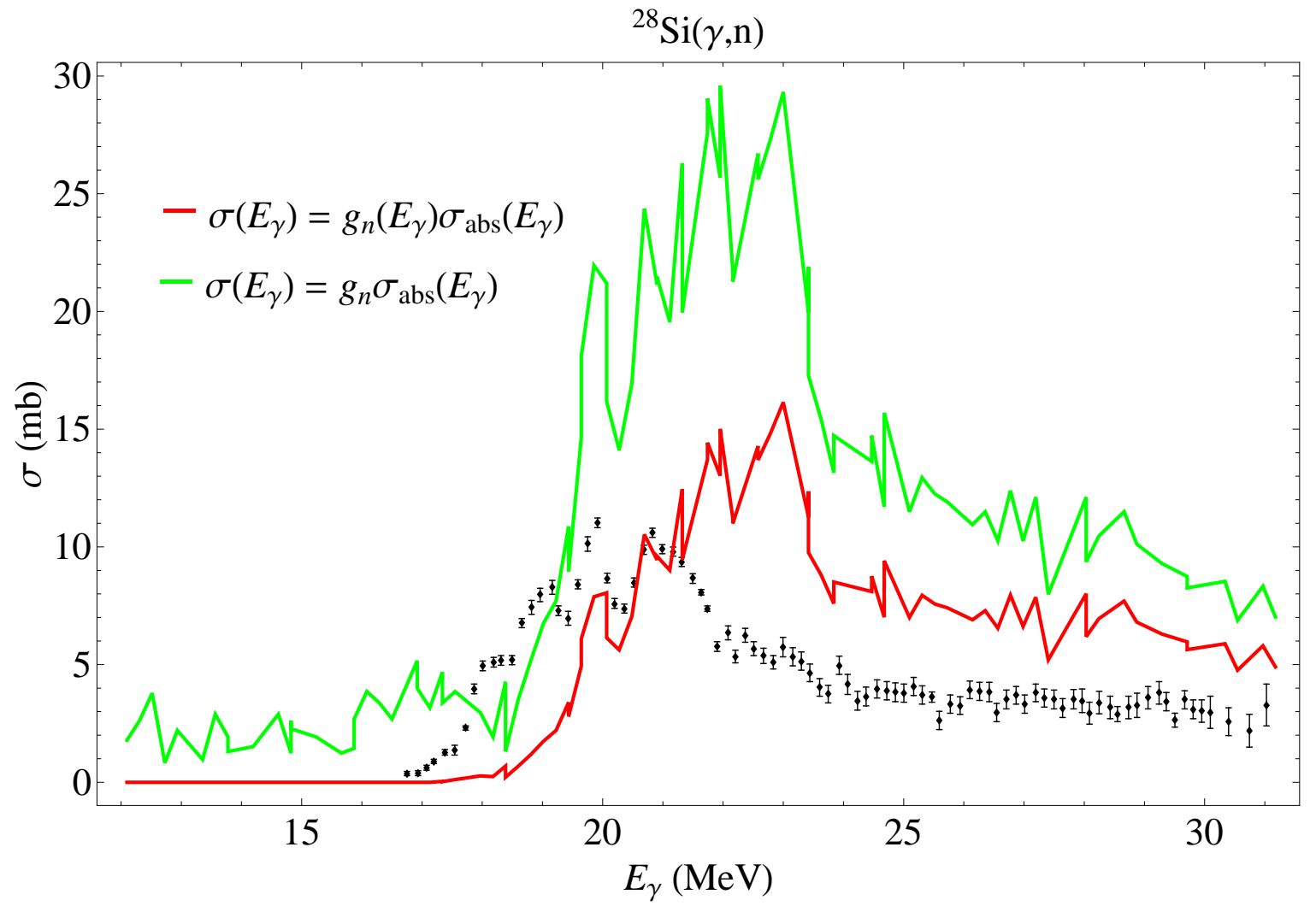

FIG. 2: Same as Figure 1, except for ${ }^{28} \mathrm{Si}$. Experimental data are from Figure 15(b) of reference $[27]$. 


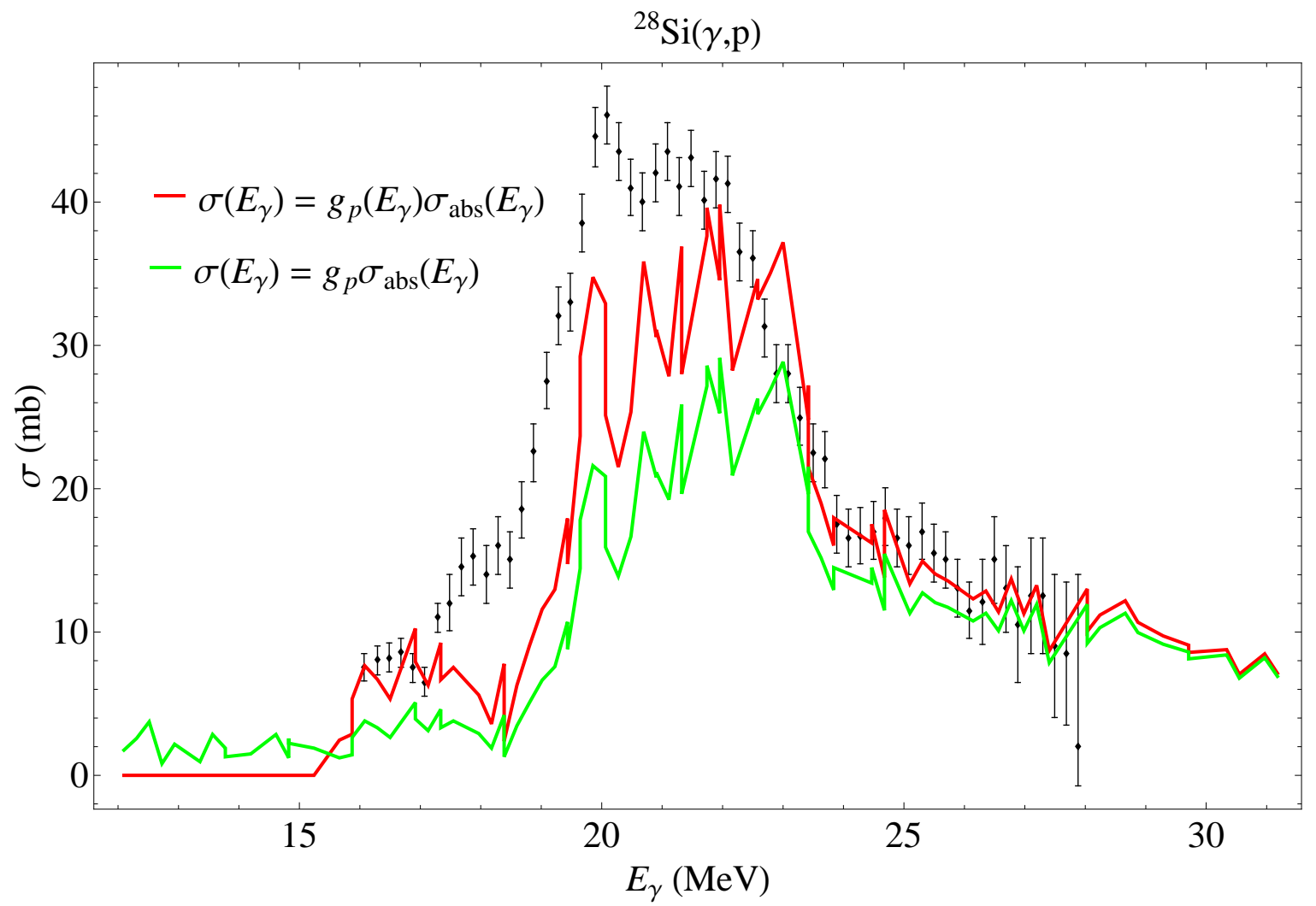

FIG. 3: Same as Figure 1, except for the photoproton cross section for ${ }^{28} \mathrm{Si}$. Experimental data are from reference [19] (p. 110). 


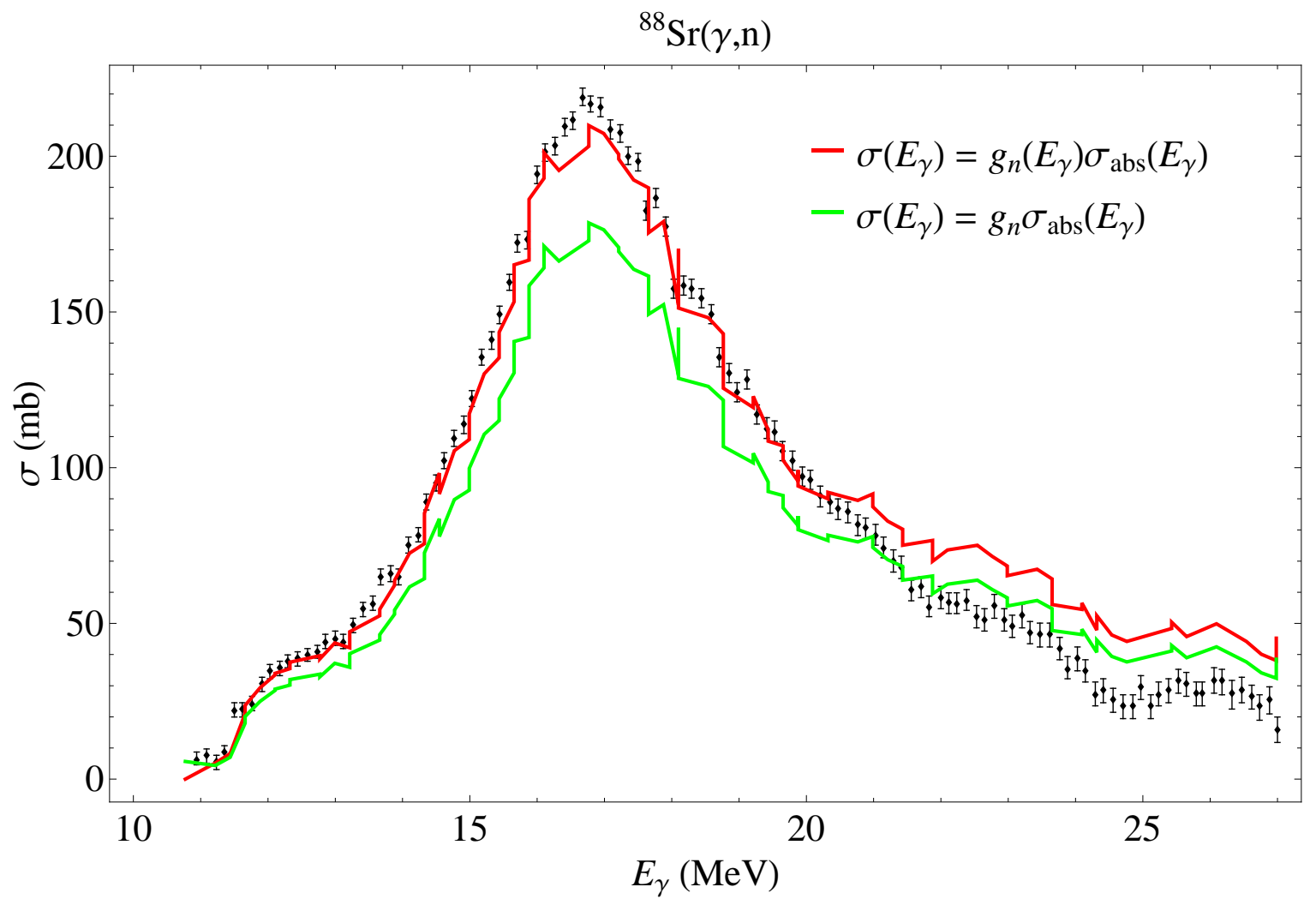

FIG. 4: Same as Figure 1, except for ${ }^{88} \mathrm{Sr}$. Experimental data are from reference [19] (p. 168). 


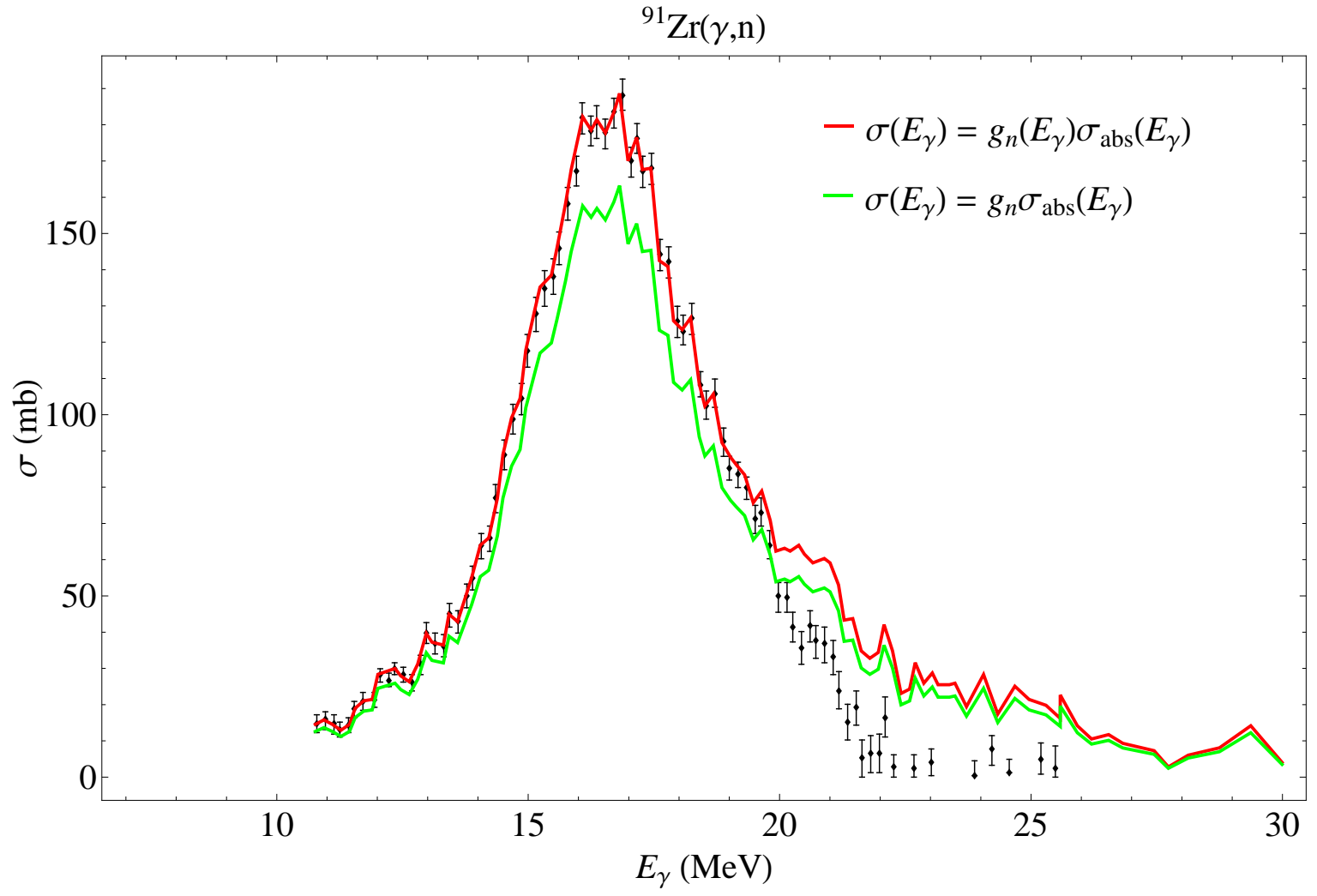

FIG. 5: Same as Figure 1, except for ${ }^{91} \mathrm{Zr}$. Experimental data are from reference [19] (p. 171). 


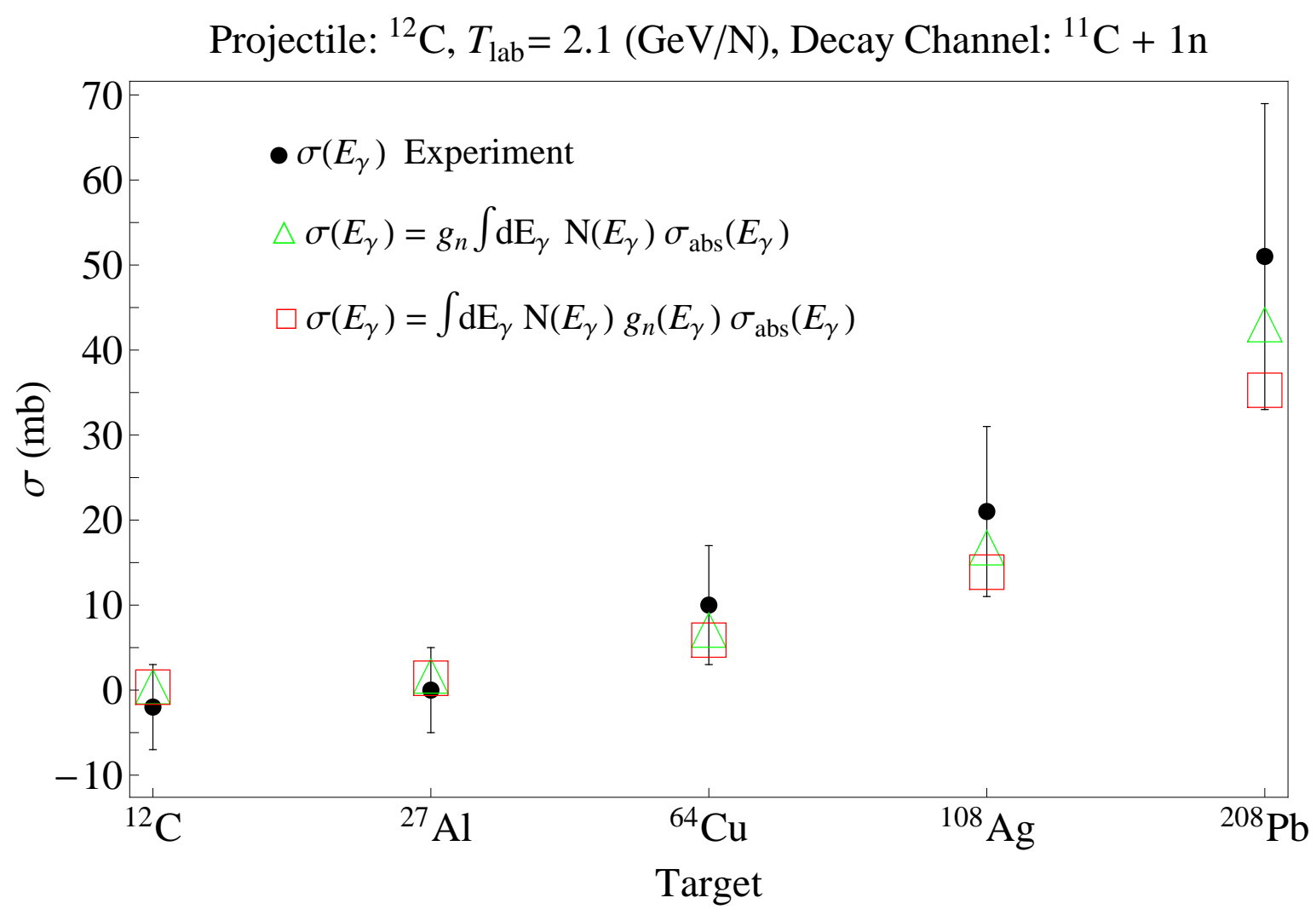

FIG. 6: Comparison between theory and experiment for the single neutron removal EMD cross section of ${ }^{12} \mathrm{C}$ for various targets at $2.1 \mathrm{GeV} / \mathrm{N}$. Experimental data [displayed as black circles] are from reference [32]. Theoretical cross sections are calculated using WE [displayed as red squares, using equation (23)] and energy independent [displayed as green triangles, using equation (24)] branching ratios. Note that this is a graphical representation of the data in Table III. 


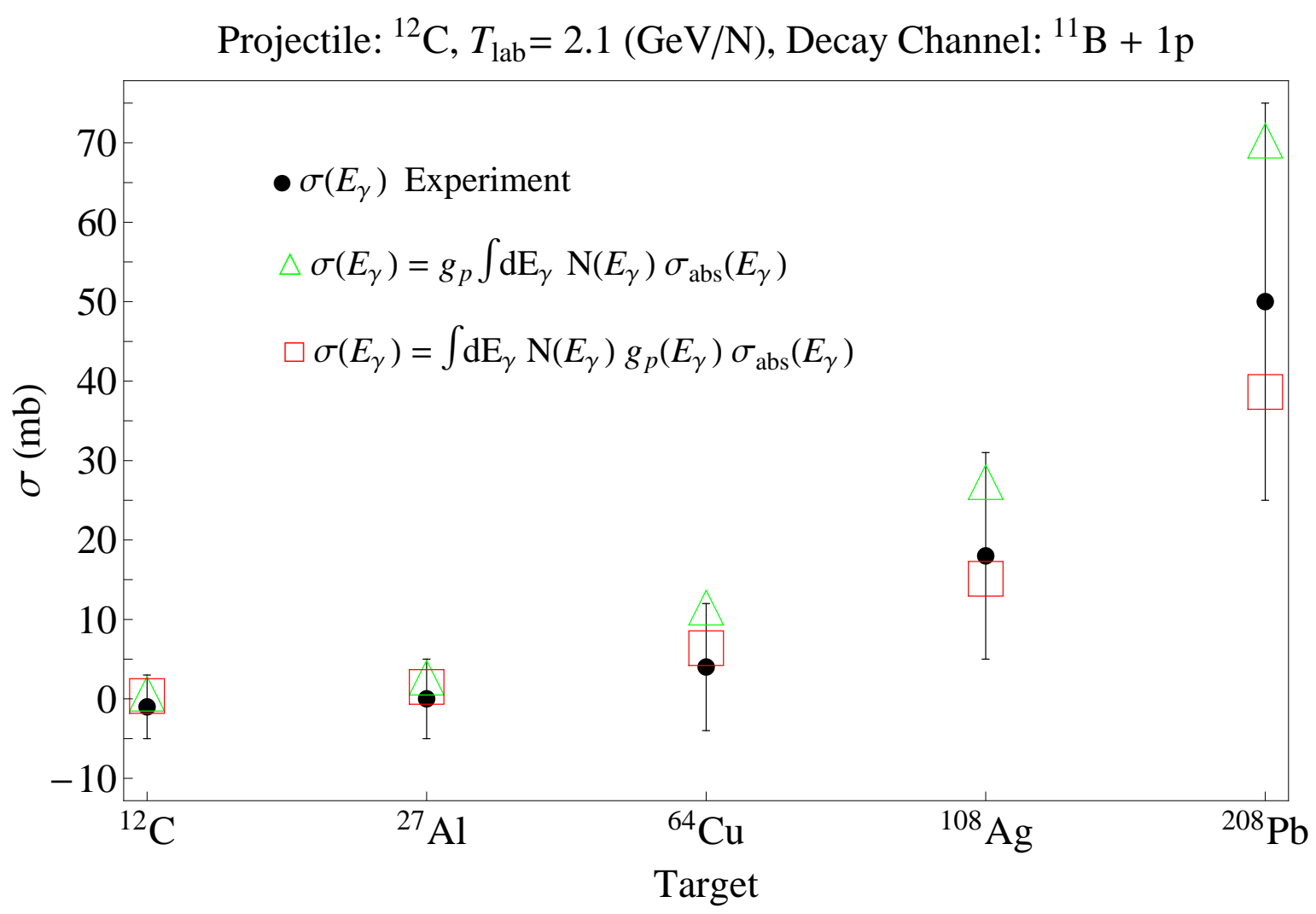

FIG. 7: Same as Figure 6, except for single proton removal. 


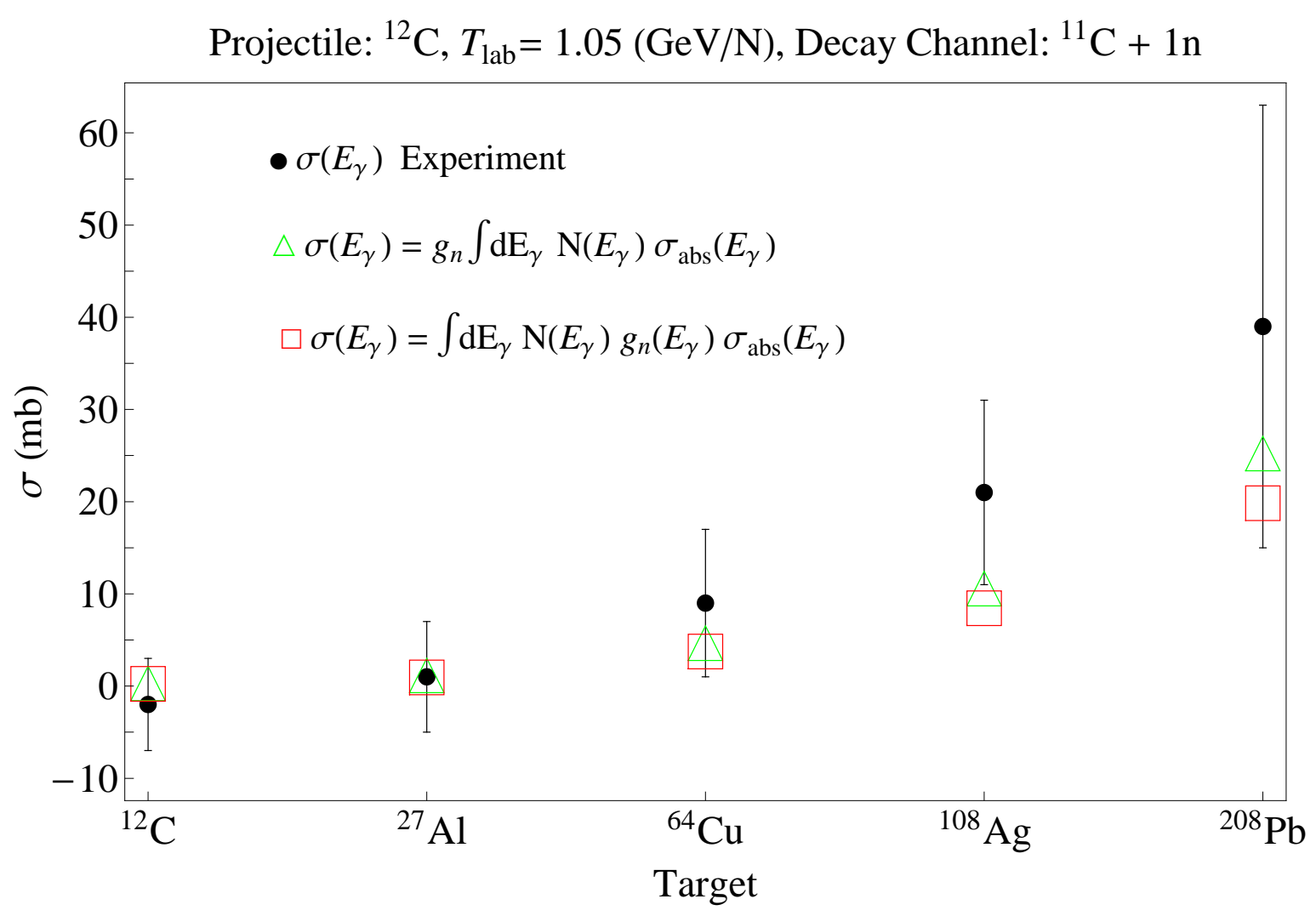

FIG. 8: Same as Figure 6, except at $1.05 \mathrm{GeV} / \mathrm{N}$. 


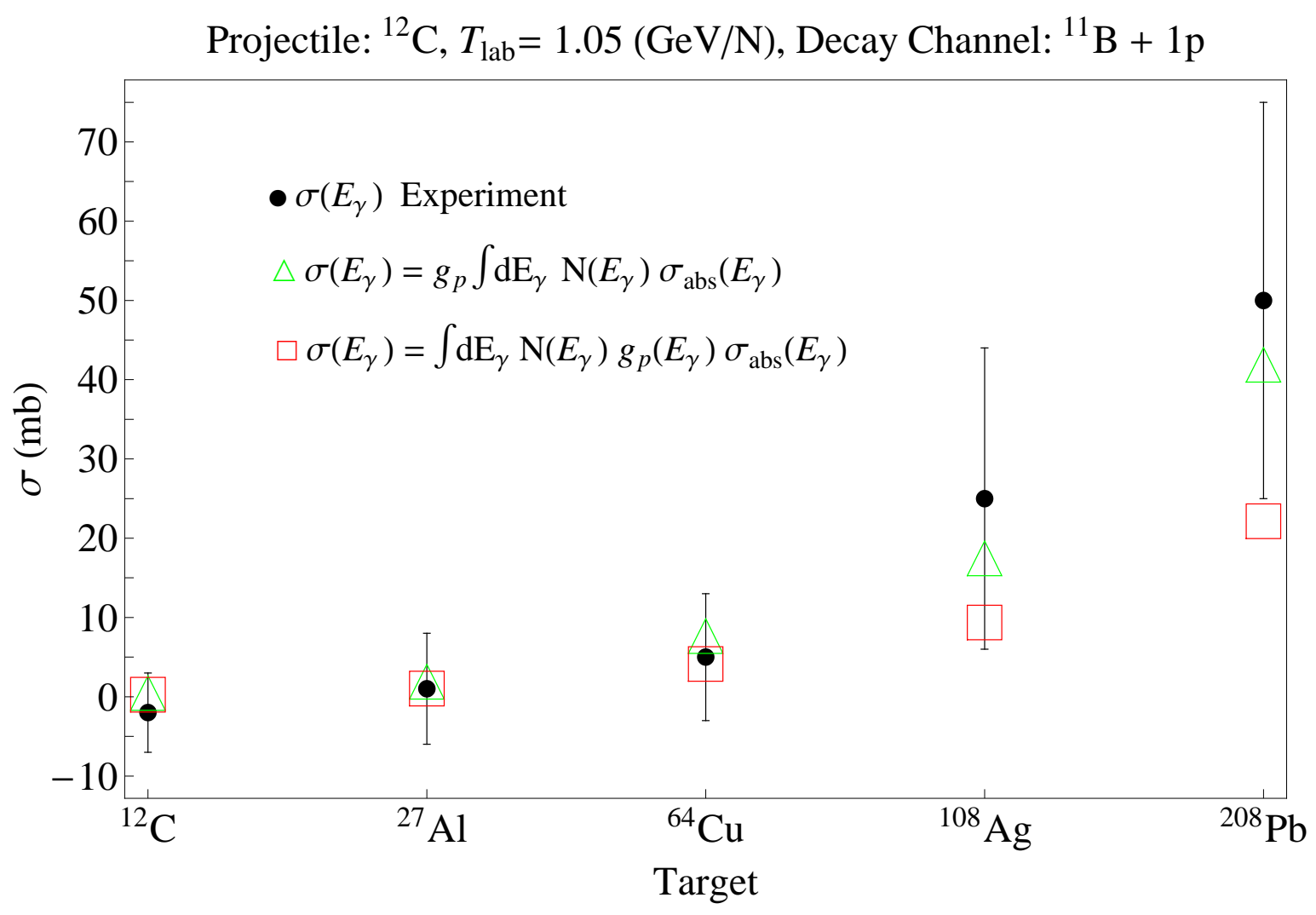

FIG. 9: Same as Figure 6, except for single proton removal at $1.05 \mathrm{GeV} / \mathrm{N}$. 


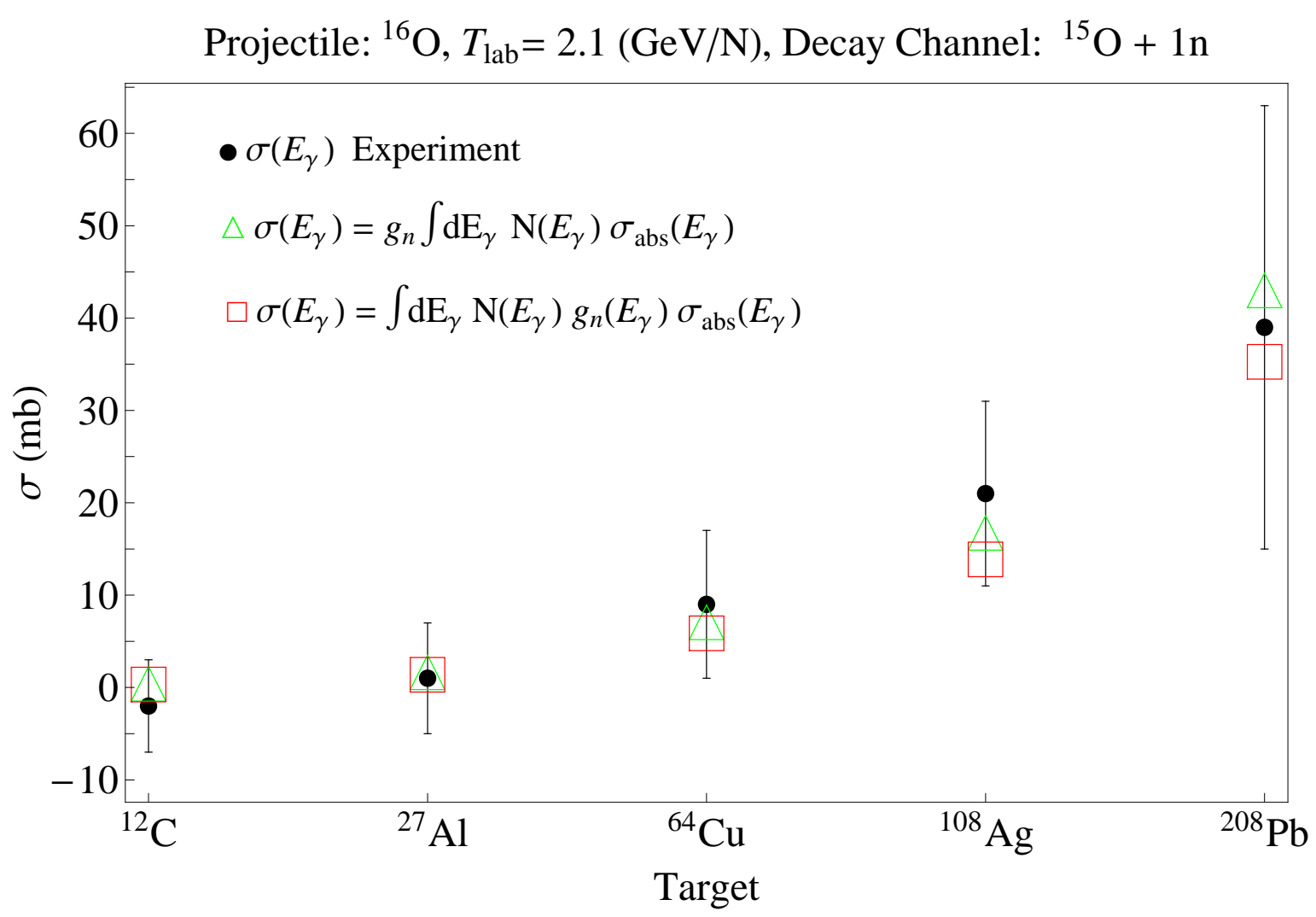

FIG. 10: Same as Figure 6, except for ${ }^{16} \mathrm{O}$ at $2.1 \mathrm{GeV} / \mathrm{N}$. 


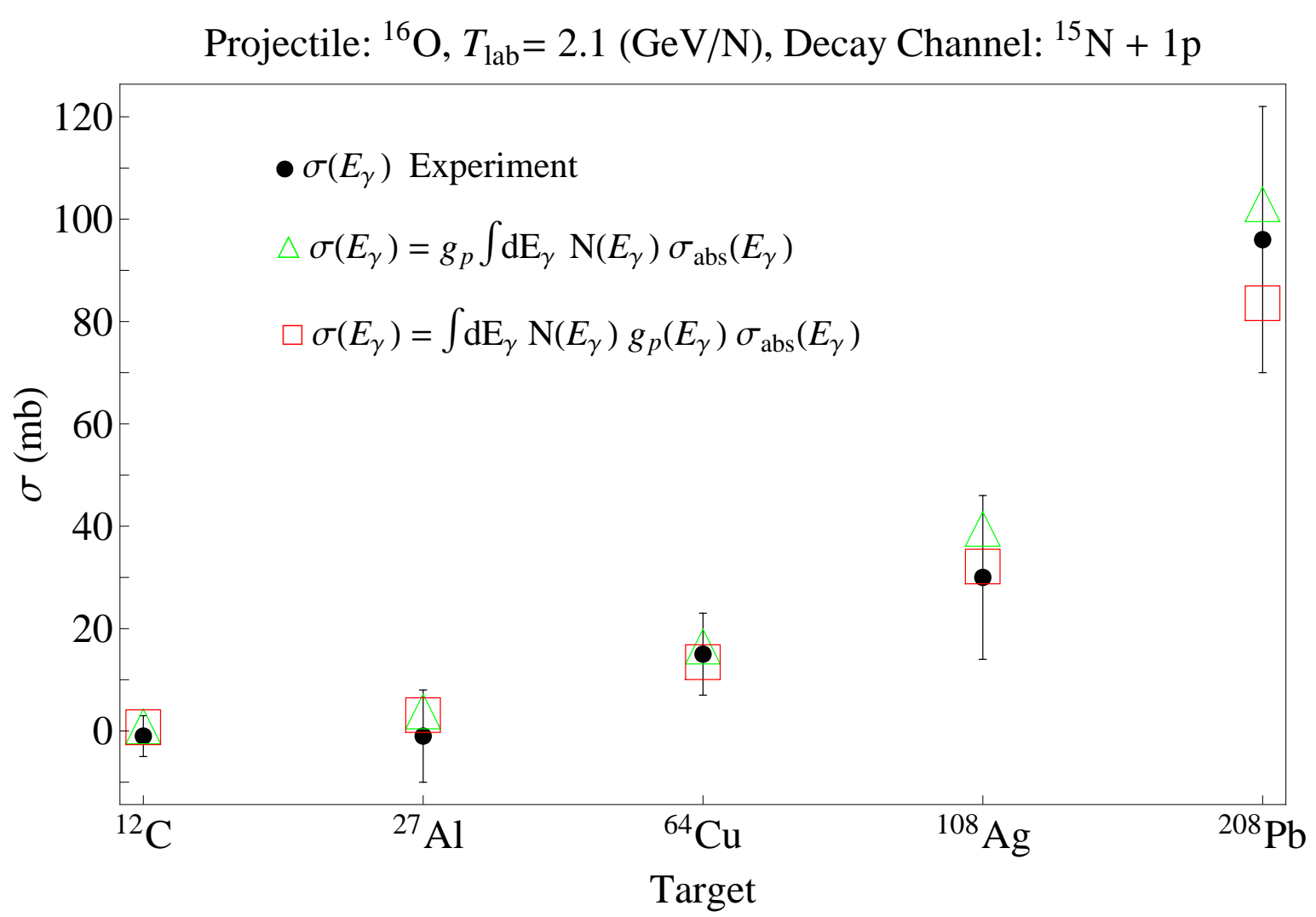

FIG. 11: Same as Figure 6, except for the single proton removal of ${ }^{16} \mathrm{O}$ at $2.1 \mathrm{GeV} / \mathrm{N}$. 


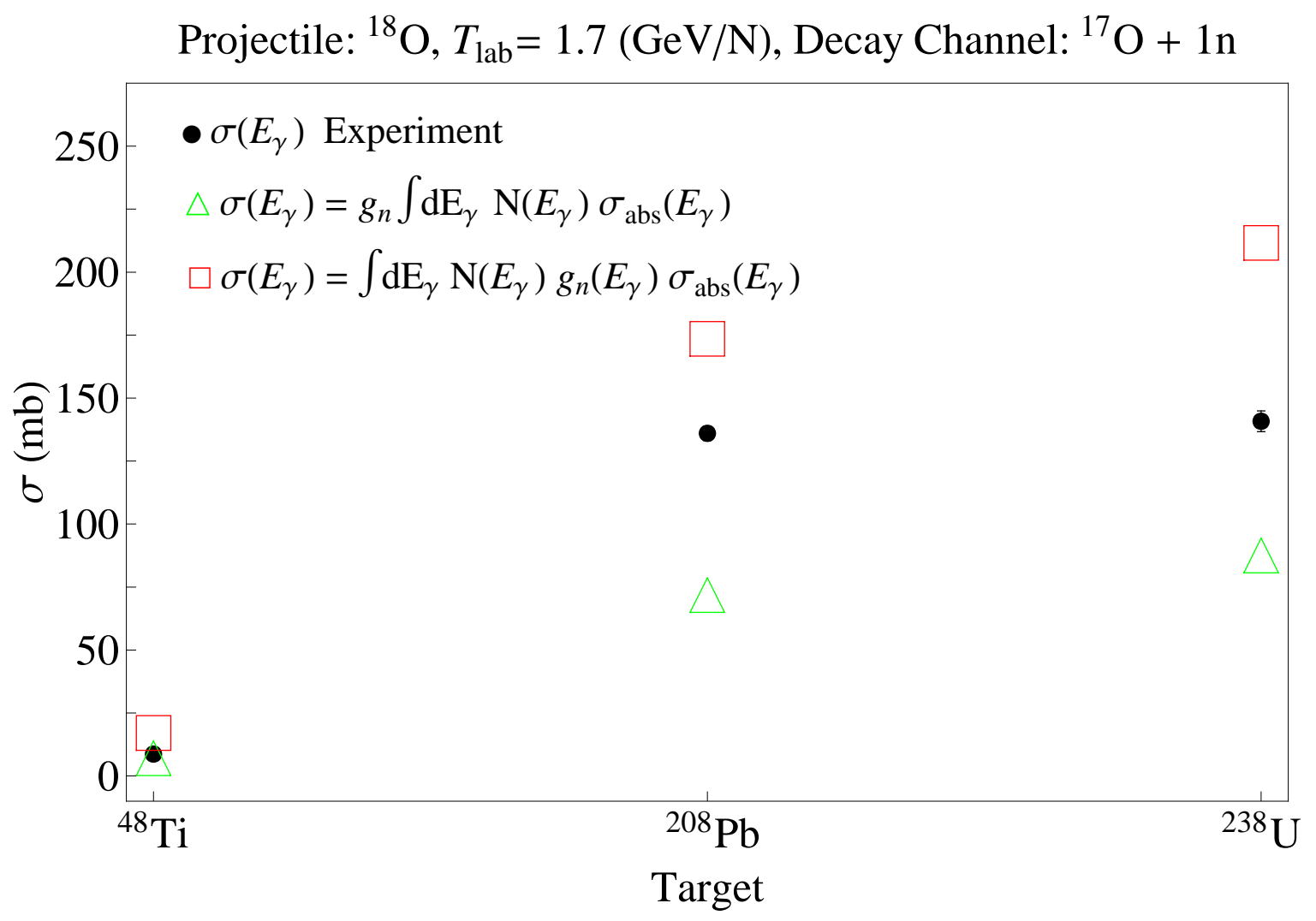

FIG. 12: Same as Figure 6, except for ${ }^{18} \mathrm{O}$ at $1.7 \mathrm{GeV} / \mathrm{N}$. Experimental data [displayed as black circles] are from reference [34]. 


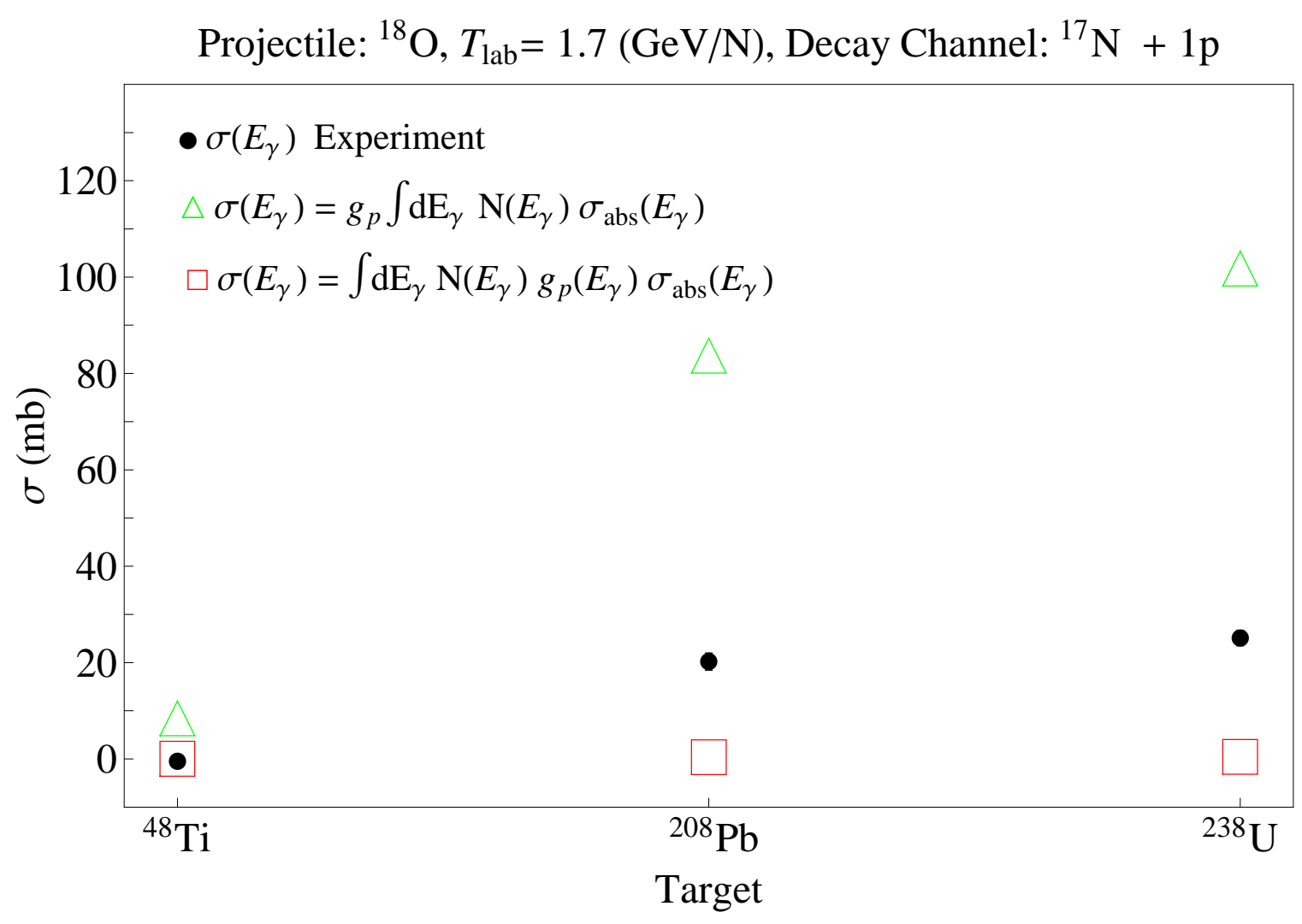

FIG. 13: Same as Figure 6, except for the single proton removal of ${ }^{18} \mathrm{O}$ at $1.7 \mathrm{GeV} / \mathrm{N}$. Experimental data [displayed as black circles] are from reference [34]. 
Projectile: ${ }^{28} \mathrm{Si}, T_{\mathrm{lab}}=14.6(\mathrm{GeV} / \mathrm{N})$, Decay Channel: ${ }^{27} \mathrm{Si}+1 \mathrm{n}$

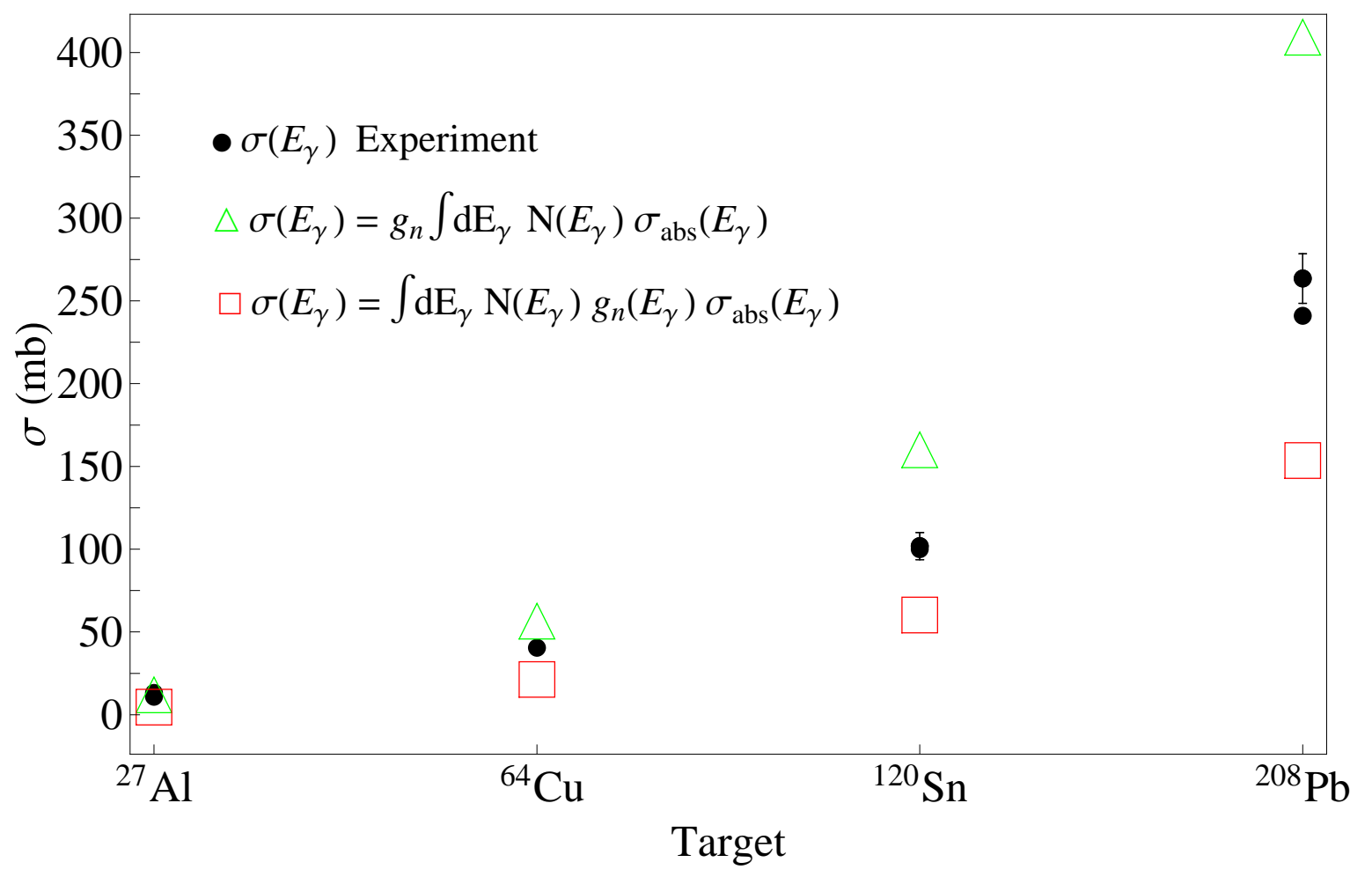

FIG. 14: Same as Figure 6, except for ${ }^{28} \mathrm{Si}$ at $14.6 \mathrm{GeV} / \mathrm{N}$. Experimental data [displayed as black circles] are from references [30, 31, 37]. 


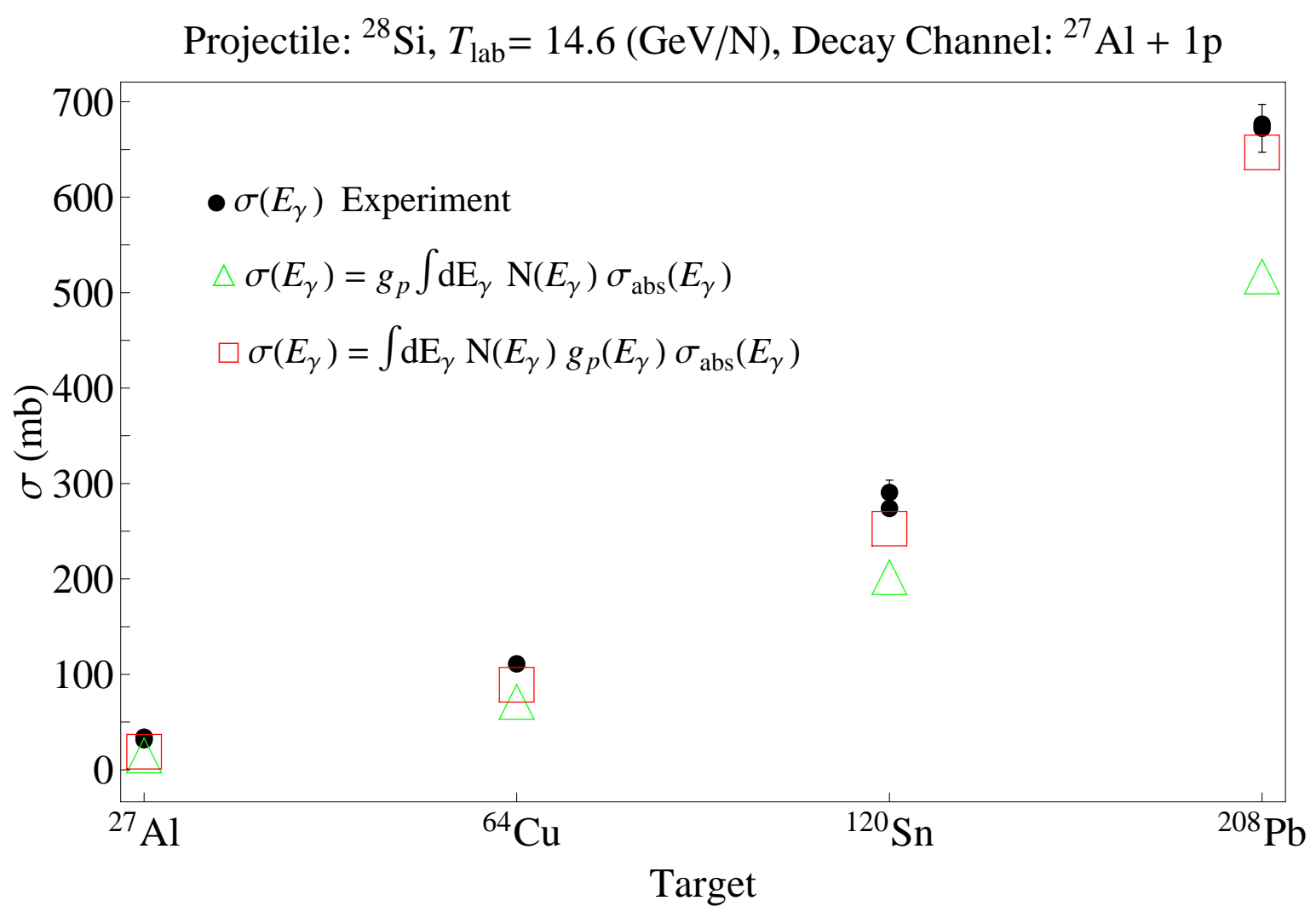

FIG. 15: Same as Figure 6, except for single proton decay of ${ }^{28} \mathrm{Si}$ at $14.6 \mathrm{GeV} / \mathrm{N}$. Experimental data [displayed as black circles] are from references [30, 31, 37]. 


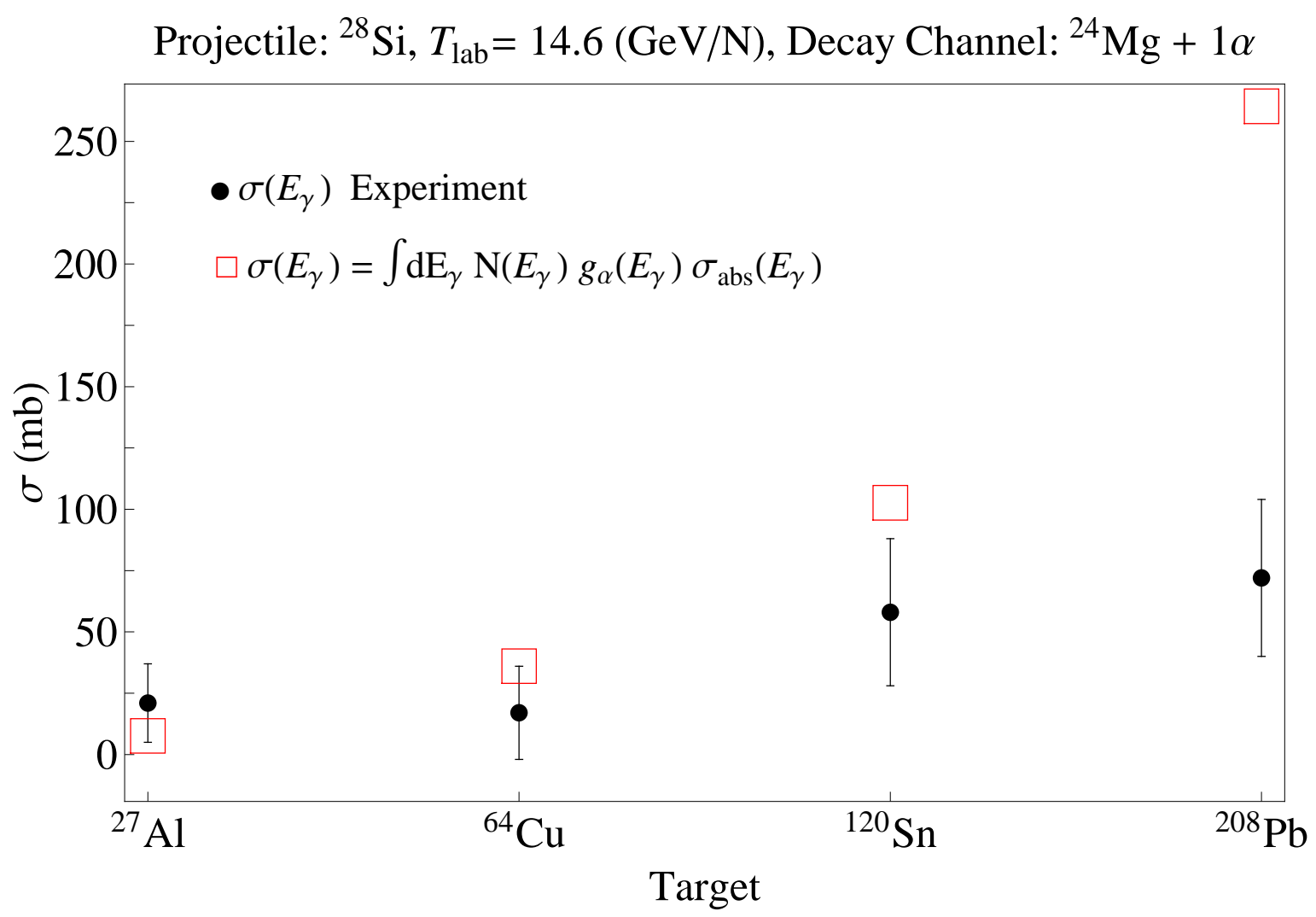

FIG. 16: Same as Figure 6, except for single alpha particle decay of ${ }^{28} \mathrm{Si}$ at $14.6 \mathrm{GeV} / \mathrm{N}$. Experimental data [displayed as black circles] are from reference [30]. Note that there are no theoretical cross sections calculated with an energy independent branching ratio since an energy independent branching ratio has not been formulated for alpha particle removal. 


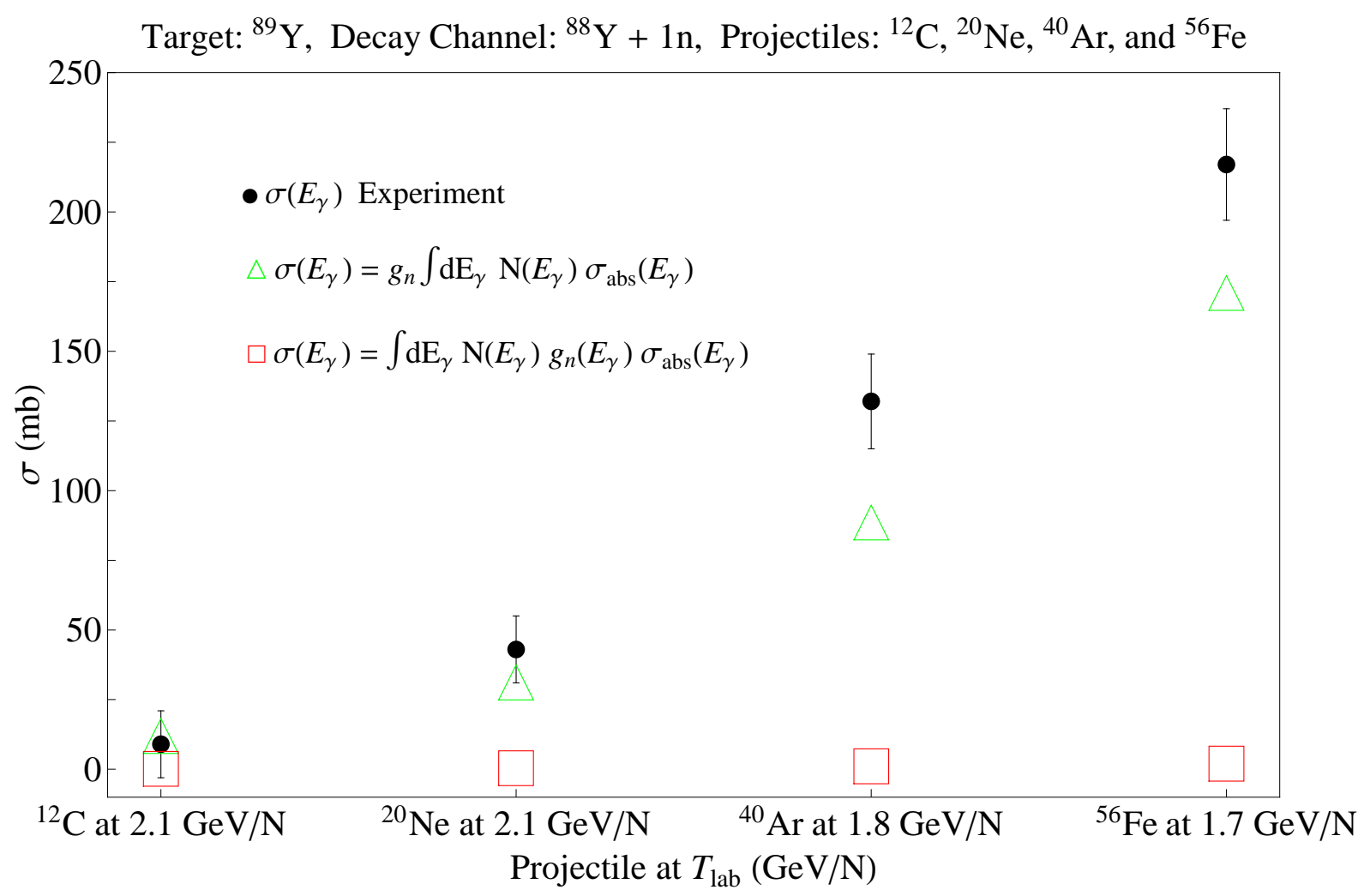

FIG. 17: Comparison between theory and experiment for the single neutron removal EMD cross section of a ${ }^{89} \mathrm{Y}$ target for various projectile and energy combinations. Note that this is a graphical representation of the data in Table III. Experimental data [displayed as black circles] are from reference [35]. Theoretical cross sections are calculated using WE [displayed as red squares, using equation (23)] and energy independent [displayed as green triangles, using equation (24)] branching ratios. 


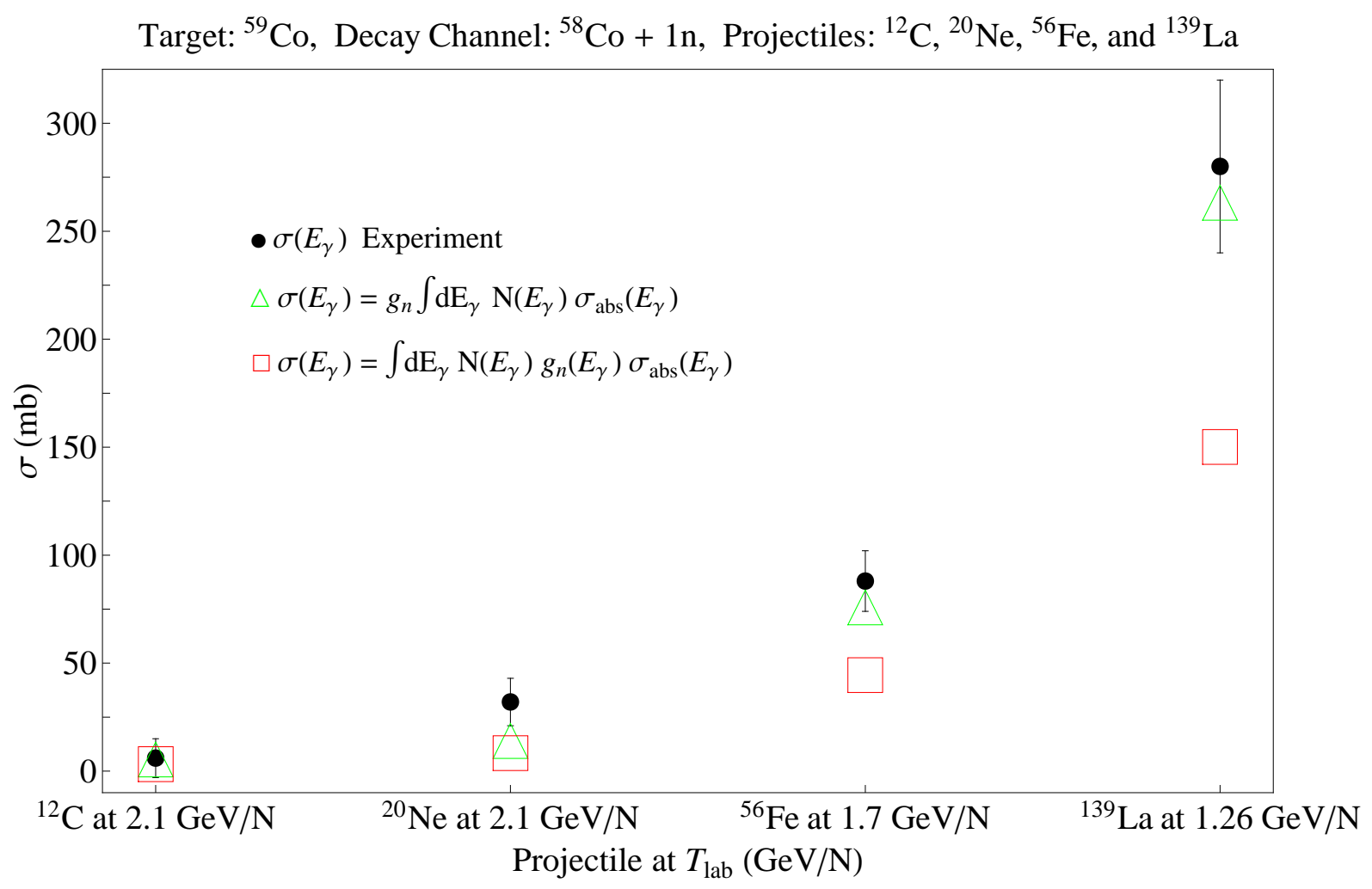

FIG. 18: Same as Figure 17, except for a ${ }^{59}$ Co target for various projectile and energy combinations. 


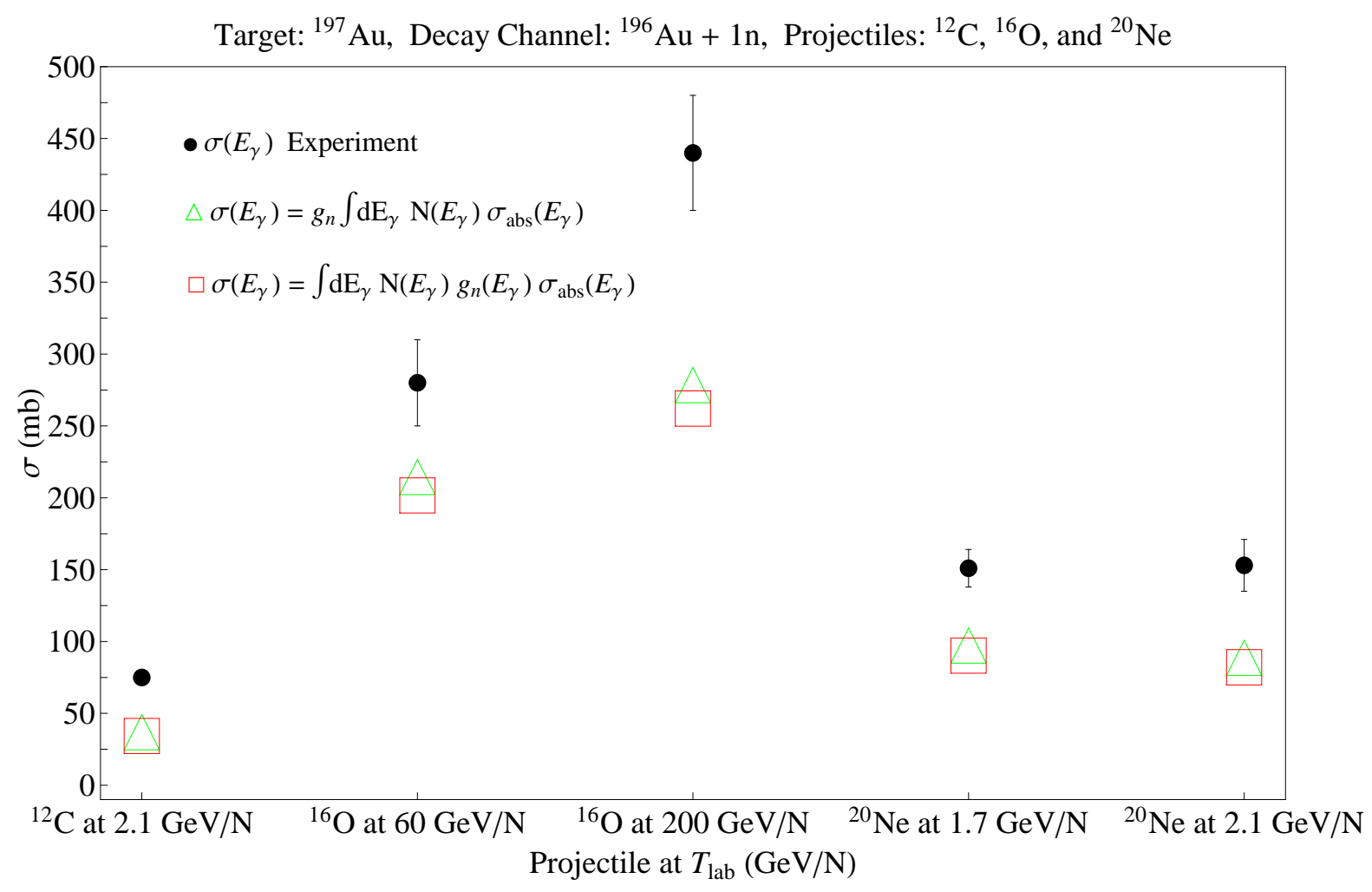

FIG. 19: Same as Figure 17, except for a ${ }^{197} \mathrm{Au}$ target for various projectile and energy combinations. Experimental data [displayed as black circles] are from references [36] and [35]. 


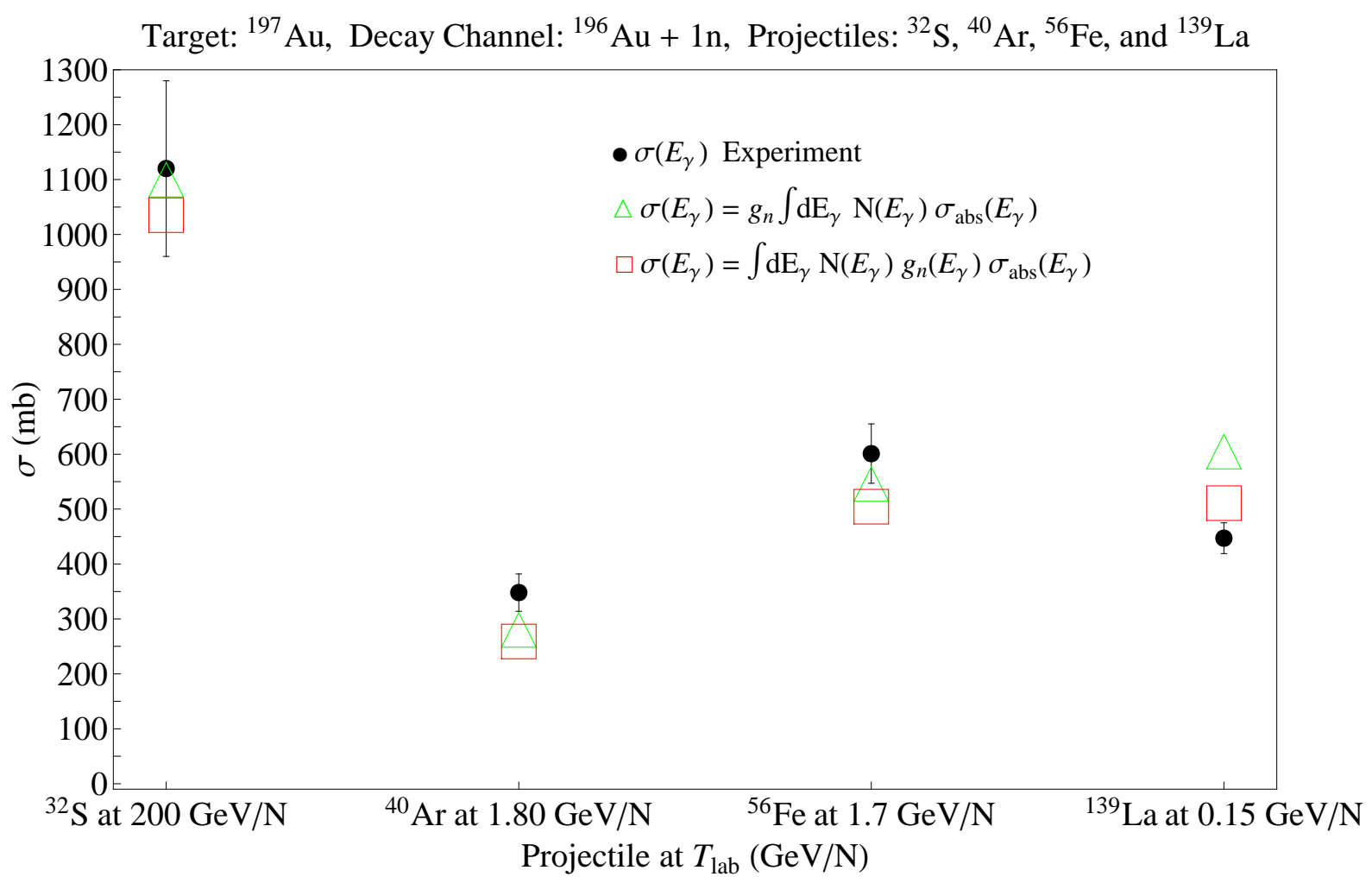

FIG. 20: Same as Figure 17, except for a ${ }^{197} \mathrm{Au}$ target for various projectile and energy combinations. Experimental data [displayed as black circles] are from references [36] and [35]. 
[1] J. Wilson, R. Tripathi, F. Badavi, and F. Cucinotta, in Proceedings of the 36th Conference on Environmental Systems (ICES) (Norfolk, Virginia, 2006).

[2] J. Wilson, R. Tripathi, G. Qualls, F. Cucinotta, R. Prael, J. Norbury, J. Heinbockel, and J. Tweed, Adv. Space Res. 34, 1328 (2004).

[3] J. Wilson, R. Tripathi, F. Cucinotta, J. S. F. Badavi, S. C. J. Norbury, C. Zeitlin, L. Heilbronn, and J. Miller, Tech. Rep. TP-3533, NASA (1995).

[4] P. Frobrich and R. Lipperheide, Theory of Nuclear Reactions (Clarendon Press, Oxford, 1996).

[5] J. Norbury and L. Townsend, Tech. Rep. TP-2527, NASA (1986).

[6] C. Bertulani and P. Danielewicz, Introduction to Nuclear Reactions (Institute of Physics Publishing, Bristol, 2004).

[7] J. Newton, Phys. Scr. 24, 83 (1981).

[8] W. Stephens, Nuclear Fission and Atomic Energy (Inman, Pennsylvania, 2008).

[9] A. Iljinov, M. Kazarnovsky, and E. Paryev, Intermediate-Energy Nuclear Physics (AddisonWesley, Boca Raton, 1994).

[10] E. Cherepanov, A. Ilijinov, and M. Mebel, J. Phys. G: Nucl. Phys 9, 1397 (1983).

[11] I. Dostrovsky, Z. Fraenkel, and G. Friedlander, Phys. Rev. 116, 683 (1959).

[12] A. Adamczyk and J. Norbury, Photonuclear cross sections using weisskopf-ewing and hauserfeshbach theory (2010), submitted to Nuclear Instruments and Methods B.

[13] A. Adamczyk, Electromagnetic nucleus-nucleus cross sections using energy dependent branching ratios (2009), Ph.D. Dissertation, Worcester Polytechnic Institute, Worcester, MA.

[14] G. Westfall, L. Wilson, P. Lindstrom, H. Crawford, D. Greiner, and H. Heckman, Phys. Rev. C 19, 1309 (1979).

[15] J. Norbury and L. Townsend, Astrophys. J. 86, 307 (1993), ISSN 0067-0049.

[16] J. Jackson, Classical Electrodynamics (Wiley, New Jersey, 1999), 3rd ed.

[17] C. Bertulani and G. Baur, Phys. Rep. 163, 299 (1988), ISSN 0370-1573.

[18] J. Norbury, Phys. Rev. C 40, 2621 (1989).

[19] Handbook on photonuclear data for applications - Cross sections and spectra, IAEA, tecdocdraft no. 3 ed. (2000).

[20] D. Brink, ed., Workshop in Chaos and Collectivity in Many Body Systems (Dresden, 2008).

[21] J. Speth, Electric and Magnetic Giant Resonances in Nuclei, vol. 7 of International Review of Nuclear Physics (World Scientific, New Jersey, 1991).

[22] Y. Abgrall, G. Baron, E. Caurier, and G. Monsonego, Nucl. Phys. A 131, 609 (1969), ISSN 0375-9474.

[23] E. Van Camp, R. Van de Vyver, E. Kerkhove, D. Ryckbosch, H. Ferdinande, P. Van Otten, and P. Berkvens, Phys. Rev. C 24, 2499 (1981).

[24] E. Van Camp, D. Ryckbosch, R. Van de Vyver, E. Kerkhove, P. Van Otten, and P. Berkvens, Phys. Rev. C 30, 1182 (1984).

[25] H. Bartsch, K. Huber, U. Kneissl, and H. Krieger, Nucl. Phys. A 256, 243 (1976).

[26] C. Benesh, Phys. Rev. C 46, 2635 (1992).

[27] B. Berman and S. Fultz, Rev. Mod. Phys. 47, 713 (1975).

[28] C. D. Jager, H. D. Vries, and C. D. Vries, At. Data and Nucl. Data Tables 14, 479 (1974).

[29] H. D. Vries, C. D. Jager, and C. D. Vries, At. Data and Nucl. Data Tables 36, 495 (1987).

[30] J. Barrette, R. Bellwied, P. Braun-Munzinger, W. E. Cleland, G. David, J. Dee, O. Dietzsch, 
E. Duek, M. Fatyga, D. Fox, et al., Phys. Rev. C 51, 865 (1995).

[31] J. Barrette, P. Braun-Munzinger, W. E. Cleland, G. David, E. Duek, M. Fatyga, D. Fox, S. V. Greene, J. R. Hall, R. Heifetz, et al., Phys. Rev. C 41, 1512 (1990).

[32] H. Heckman and P. Lindstrom, Phys. Rev. Lett. 37, 56 (1976).

[33] W. V. Oz and M. Yannakakis, eds., Proc. 23rd Recontre de Moriond Current Issues in Hadron Physics, no. 147 in ed. J. Tran. Thanh Van, (gif - sur - Yvette: Editions Frontieres) (1988).

[34] D. Olson, B. Berman, D. Greiner, H. Heckman, P. Lindstrom, G. Westfall, and H. Crawford, Phys. Rev. C 24, 1529 (1981).

[35] J. W. Norbury, Phys. Rev. C 47, 406 (1993).

[36] T. Aumann, J. V. Kratz, E. Stiel, K. Sümmerer, W. Brüchle, M. Schädel, G. Wirth, M. Fauerbach, and J. C. Hill, Phys. Rev. C 47, 1728 (1993).

[37] W. J. Llope and P. Braun-Munzinger, Phys. Rev. C 41, 2644 (1990). 